\title{
Competition between Intramolecular and Intermolecular C-H Activation in Iridium Ethene Complexes
}

\author{
Tanachat W. Bell, Sally-Ann Brough, Martin G. Partridge, Robin N. Perutz," and \\ A. Denise Rooney* \\ Department of Chemistry, University of York, York YO1 5DD, U.K.
}

Received February 25, 1993

\begin{abstract}
The ethene complexes $\mathrm{CpIr}(\mathrm{L})\left(\mathrm{C}_{2} \mathrm{H}_{4}\right)\left(\mathrm{Cp}=\eta^{5}-\mathrm{C}_{5} \mathrm{H}_{5}, \mathrm{~L}=\mathrm{PPh}_{3}, \mathrm{CO}\right)$ undergo two competing photochemical reactions in solution: (i) isomerization to the vinyl hydride $\mathrm{CpIr}(\mathrm{L})\left(\mathrm{C}_{2} \mathrm{H}_{3}\right) \mathrm{H}$ and (ii) dissociation of ethene and insertion into solvent $\mathrm{C}-\mathrm{H}$ bonds to form $\mathrm{Cp} \operatorname{Ir}(\mathrm{L})(\mathrm{R}) \mathrm{H}$. The vinyl hydride is favored over the intermolecular product by longer wavelength photolysis, lower temperature, and a more rigid surrounding medium and by $\mathrm{L}=\mathrm{PPh}_{3}$ compared to $\mathrm{L}=\mathrm{CO}$. The vinyl hydride isomer is the exclusive product of $\mathrm{UV}$ irradiation of $\operatorname{CpIr}(\mathrm{CO})\left(\mathrm{C}_{2} \mathrm{H}_{4}\right)$ in solid toluene $(77 \mathrm{~K})$ or solid argon $(12 \mathrm{~K})$ but competes with formation of $\mathrm{CpIr}(\mathrm{CO})_{2}$ in $\mathrm{CO}$-doped Ar matrices and $\mathrm{CpIr}(\mathrm{CO})\left(\mathrm{CH}_{3}\right) \mathrm{H}$ in methane matrices. The latter is also formed by secondary photolysis of the vinyl hydride. The barrier for the thermal conversion of $\mathrm{CpIr}(\mathrm{L})\left(\mathrm{C}_{2} \mathrm{H}_{3}\right) \mathrm{H}$ to the parent ethene complex $\operatorname{CpIr}(\mathrm{L})\left(\mathrm{C}_{2} \mathrm{H}_{4}\right)$ increases in the following order: $\mathrm{L}=\mathrm{C}_{2} \mathrm{H}_{4}, \Delta H^{*}=$ $67 \pm 5 \mathrm{~kJ} \mathrm{~mol}^{-1} ; \mathrm{L}=\mathrm{CO}, \Delta H^{*}=95 \pm 4 \mathrm{~kJ} \mathrm{~mol}^{-1} ; \mathrm{L}=\mathrm{PPh}_{3}, \Delta G^{*}=135 \pm 3 \mathrm{~kJ} \mathrm{~mol}^{-1}$ at $318 \mathrm{~K}$. These findings are consistent with our proposal that $\mathrm{C}-\mathrm{H}$ bond activation occurs via a "cagecomplex" intermediate. However, the marked influence of temperature on the reaction indicates that intermolecular $\mathrm{C}-\mathrm{H}$ bond activation involves a thermally activated step. The photochemical reaction of $\mathrm{CpIr}\left(\mathrm{C}_{2} \mathrm{H}_{4}\right)_{2}$ with $\mathrm{PPh}_{3}$ in $\mathrm{CD}_{3} \mathrm{CN}$ generates the vinyl hydride $\mathrm{CpIr}\left(\mathrm{PPh}_{3}\right)\left(\mathrm{C}_{2} \mathrm{H}_{3}\right) \mathrm{H}$ via $\mathrm{CpIr}\left(\mathrm{PPh}_{3}\right)\left(\mathrm{C}_{2} \mathrm{H}_{4}\right)$. The corresponding reaction occurs in benzene with the additional formation of the solvent activation product $\mathrm{CpIr}\left(\mathrm{PPh}_{3}\right)(\mathrm{Ph}) \mathrm{H}$.
\end{abstract}

\section{Introduction}

The photoinduced isomerization of metal ethene to metal vinyl hydride complexes has proved to be a widespread phenomenon, especially among complexes of second- and third-row transition metals. ${ }^{1-8}$ The metal vinyl hydrides are usually thermally unstable with respect to the precursor complexes (eq 1), but the temperature

$$
|M|-\| \frac{\mathrm{hv}}{\Delta}|M|^{\mathrm{H}}
$$

required for this back-reaction varies according to the metal and ancillary ligands. The span of temperatures necessary to induce the back-reaction is surprisingly large, varying from $-20^{\circ} \mathrm{C}$ for $[\mathrm{M}]=\mathrm{CpRh}\left(\mathrm{PMe}_{3}\right)$ to $+180^{\circ} \mathrm{C}$ for $[\mathrm{M}]=\mathrm{Cp} * \operatorname{Ir}\left(\mathrm{PMe}_{3}\right)\left(\mathrm{Cp}=\eta^{5}-\mathrm{C}_{5} \mathrm{H}_{5}, \mathrm{Cp} *=\eta^{5}-\mathrm{C}_{5} \mathrm{Me}_{5}\right)$. For some tris(pyrazolyl)borate complexes, the relative stabilities of the ethene complex and the vinyl hydride complex are reversed, so that the forward reaction of eq 1 may be achieved thermally as well as photochemically. ${ }^{9,10}$ It has been postulated that the increased thermal stability of these vinyl hydride complexes is associated with

(1) Stoutland, P. O.; Bergman, R. G. J. Am. Chem. Soc. 1988, 110, 5732.

(2) Haddleton, D. M.; Perutz, R. N. J. Chem. Soc., Chem. Commun. 1986, 1734 .

(3) Baker, M. V.; Field, L. D. J. Am. Chem. Soc. 1986, 108, 7433, 7436.

(4) Wenzel, T. T.; Bergman, R. G. J. Am. Chem. Soc. 1986, 108, 4856.

(5) Bell, T. W.; Haddleton, D. M.; McCamley, A.; Partridge, M. G.; Perutz, R. N.; Willner, H. J. Am. Chem. Soc. 1990, 112, 9212

(6) McCamley, A.; Perutz, R. N.; Stahl, S.; Werner, H. Angew. Chem., Int. Ed. Engl. 1989, 28, 1690.

(7) Tanke, R. S.; Crabtree, R. H. Inorg. Chem. 1989, 28, 3444.

(8) Schulz, M.; Werner, H. Organometallics 1992, 11, 2790

(9) Ghosh, C. K.; Hoyano, J. K.; Krentz, R.; Graham, W. A. G. J. Am. Chem. Soc. 1989, 111,5480 .

(10) Perez, P. J.; Poveda, M. L.; Carmona, E. J. Chem. Soc., Chem. Commun. 1992, 8.
Scheme I. Cage-Complex Mechanism for the Isomerization of the Ethene Complexes

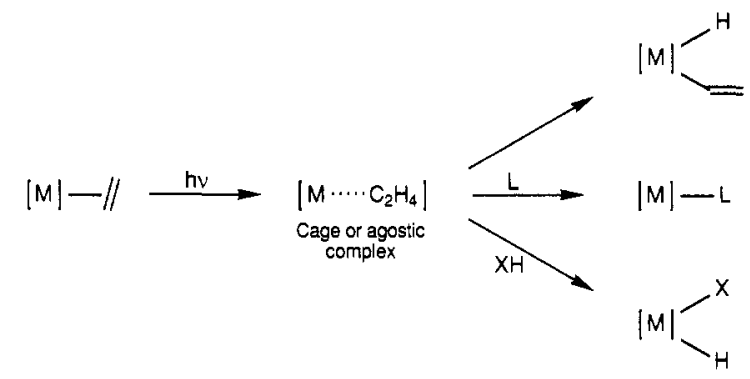

conversion of the tris(pyrazolyl)borate ligand from $\eta^{2}$ - to $\eta^{3}$-coordination.

In a previous paper, we proposed that the photoisomerization reactions proceed via cage complexes between metal and expelled ethene (Scheme I). ${ }^{5}$ The extent of interaction within the cage is postulated to govern the proportion of photoisomerization compared to reaction with solvents or added ligands. When the interaction in the cage is strong, photoisomerization is dominant and may be observed at room temperature in preference to solvent activation (e.g. for $\left.\mathrm{CpIr}\left(\mathrm{PMe}_{3}\right)\left(\mathrm{C}_{2} \mathrm{H}_{4}\right)\right)$. When the interaction is weak, as for $\mathrm{CpRh}\left(\mathrm{PMe}_{3}\right)\left(\mathrm{C}_{2} \mathrm{H}_{4}\right)$ or $\mathrm{CpIr}\left(\mathrm{C}_{2} \mathrm{H}_{4}\right)_{2}$, photoisomerization is only significant in solid matrices such as toluene or argon at low temperature. The isomerization of $\left(\eta^{6}\right.$. mesitylene) $\mathrm{Os}\left(\mathrm{C}_{2} \mathrm{H}_{4}\right) \mathrm{CO}$ represents an intermediate case. ${ }^{6}$ Thus there is a gradation of "intramolecularity" between one complex and another. The order of increasing intramolecularity correlates with the order of stability of the vinyl hydride complexes with respect to metal ethene complexes. Our postulate of the cage complex is equivalent to Bergman's " $\sigma$-complex" formed as an intermediate in the thermal reaction of $\mathrm{Cp} * \operatorname{Ir}\left(\mathrm{PMe}_{3}\right)($ cyclohexyl) $\mathrm{H}$ with ethene. ${ }^{1}$ There is one observation of such a $\sigma$-complex, 
viz. $\mathrm{Fe}\left(\eta^{1}-\mathrm{C}_{2} \mathrm{H}_{4}\right)$ observed in low temperature matrices; this complex does indeed isomerize to $\mathrm{Fe}\left(\mathrm{C}_{2} \mathrm{H}_{3}\right) \mathrm{H}$, albeit photochemically. ${ }^{11}$

An alternative mechanism for the isomerization of the $\mathrm{CpM}(\mathrm{L})\left(\mathrm{C}_{2} \mathrm{H}_{4}\right)$ complexes is that proposed by Tanke and Crabtree for (tris(pyrazolyl)borato) bis(ethylene)iridium(I). ${ }^{7}$ They postulated that the isomerization of this complex is initiated by expulsion of one of the ethene ligands. A more general version of this mechanism applied to the compounds studied here is given in eq 2 .
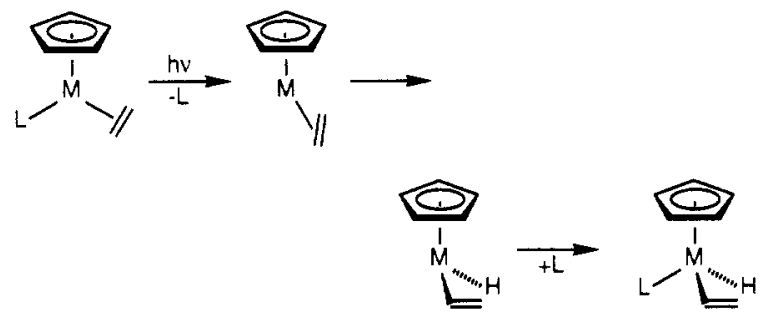

In this paper, we examine a series of reactions of $\left(\eta^{5}\right.$. cyclopentadienyl)(ethene)iridium complexes, $\operatorname{CpIr}\left(\mathrm{C}_{2} \mathrm{H}_{4}\right) \mathrm{L}$ ( $\mathrm{L}=\mathrm{CO}, \mathrm{PPh}_{3}, \mathrm{C}_{2} \mathrm{H}_{4}$ ), in which there is competition between photoisomerization and attack on other ligands or on the solvent/matrix. Since the thermal stability of the vinyl hydride complexes is of particular importance in understanding the reactions, we have also measured the activation parameters for the conversion of two of them to the corresponding ethene complexes.

\section{Experimental Section}

General Methods and Materials. The compounds were synthesized and manipulated under argon using standard Schlenk and high vacuum techniques or in a nitrogen purged glovebox. The solvents were dried by refluxing over sodium/benzophenone and then distilling under an argon atmosphere. The deuterated solvents were purchased from Goss Scientific Instruments Ltd. They were all stored under vacuum in the presence of $4-\AA$ molecular sieves, apart from benzene- $d_{6}$ which was dried by stirring over potassium/benzophenone and then distilled. The gases used for the matrices ( $\mathrm{Ar}, \mathrm{N}_{2}, \mathrm{CO}, \mathrm{CH}_{4}$ ) were $\mathrm{BOC}$ research grade $(99.999 \%$ purity). The ethene gas used in the syntheses was BOC standard grade $(99.9 \%$ purity). Iridium trichloride hydrate, triphenylcarbenium tetrafluoroborate, bromoform, and triphenylphosphine were obtained from Aldrich. Hexadecane was obtained from $\mathrm{BDH}$ and purified by standard procedures prior to use.

The irradiation of the compounds was carried out using either an ILC Technology Ltd. ILC302UV xenon lamp equipped with a UV mirror giving an output range of $240-390 \mathrm{~nm}$ or a Philips HPK $125-W$ medium pressure mercury lamp. Excess heat was removed with a water filter; wavelengths were selected with cutoff or interference filters.

Solutions were irradiated in Pyrex ampules fitted with a ptfe stopcock or in sealed NMR tubes. Photolysis of the samples in frozen toluene- $d_{8}$ was carried out by immersing a sealed NMR tube in a partially silvered dewar containing liquid nitrogen. After photolysis the samples were thawed before obtaining the NMR spectra. Photolysis between 188 and $273 \mathrm{~K}$ was carried out in NMR tubes cooled by a stream of cold $\mathrm{N}_{2}$ gas. The temperature was adjusted with a JEOL controller.

Spectroscopy. The NMR spectra were recorded with \& Bruker MSL300 spectrometer unless otherwise stated. The ${ }^{1} \mathrm{H}$ NMR chemical shifts were referenced to residual protiated solvent as follows: benzene- $d_{5} \delta=7.13$, toluene- $d_{7} \delta=2.1$, dmso- $d_{5} \delta=$ 2.5 , cyclohexane- $d_{11} \delta=1.38 \mathrm{ppm}$. The ${ }^{13} \mathrm{C}$ NMR chemical shifts

(11) Kafafi, Z. H.; Hauge, R. H.; Margrave, J. L. J. Am. Chem. Soc. $1985,107,7550$. were referenced to the solvent signals as follows: toluene- $d_{8} \delta=$ $20.1, \mathrm{CDCl}_{3} \delta=77 \mathrm{ppm} .{ }^{13} \mathrm{C}$ spectra were obtained with a standard DEPT pulse sequence. The ${ }^{31} \mathrm{P}$ NMR chemical shifts were referenced externally to $\mathrm{H}_{3} \mathrm{PO}_{4}$ at $\delta 0$. The spectra were recorded with broad band ${ }^{1} \mathrm{H}$ decoupling.

The IR spectra were recorded on either a Mattson Sirius or a Mattson Research Series FTIR spectrometer with a TGS detector and $\mathrm{KBr}$ beam splitter $\left(4000-450 \mathrm{~cm}^{-1}\right)$. The spectrometer was continuously purged with $\mathrm{CO}_{2}$-free dry air. The spectra were recorded as the average of 128 scans with $1-\mathrm{cm}^{-1}$ resolution (25K data points, $64 \mathrm{~K}$ transform points).

The matrix isolation apparatus at York has been described in detail previously. ${ }^{12} \mathrm{CpIr}(\mathrm{CO})\left(\mathrm{C}_{2} \mathrm{H}_{4}\right)$ was deposited on a $\mathrm{CsI}$ window which was cooled to $12-20 \mathrm{~K}$ by an Air Products CS202 closed-cycle Displex refrigerator. The complex was sublimed at $268 \mathrm{~K}$ onto the window from a right-angled tube concurrent with the gas stream that entered the vacuum shroud from a separate inlet.

Syntheses. $\mathrm{CpIr}\left(\mathrm{C}_{2} \mathrm{H}_{4}\right)_{2}$ was prepared by a modification of the Mayer and Calabrese synthesis..$^{5,13} \operatorname{CpIr}(\mathrm{CO})\left(\mathrm{C}_{2} \mathrm{H}_{4}\right)$ was prepared by the literature procedure. ${ }^{14} \mathrm{CpIr}\left(\mathrm{PPh}_{3}\right)\left(\mathrm{C}_{2} \mathrm{H}_{4}\right)$ was prepared via $[\mathrm{CpIrCl}]_{x}, \mathrm{CpIrCl}_{2}\left(\mathrm{PPh}_{3}\right), \mathrm{CpIr}\left(\mathrm{CH}_{3}\right)_{2}\left(\mathrm{PPh}_{3}\right)$, and $\left[\mathrm{CpIr}\left(\mathrm{PPh}_{3}\right)\left(\mathrm{C}_{2} \mathrm{H}_{4}\right) \mathrm{H}_{3} \mathrm{PF}_{6}\right.$.

$\left[\mathrm{CpIrCl}_{2}\right]_{x} . \mathrm{CpIr}\left(\mathrm{C}_{2} \mathrm{H}_{4}\right)_{2}(230 \mathrm{mg})$ was dissolved in thf (15 $\mathrm{cm}^{3}$ ). The solution was degassed and hydrogen chloride gas admitted with vigorous stirring. The solution immediately changed color to dark orange, and a red precipitate formed. The precipitate was collected and washed with thf and hexane (225 $\mathrm{mg}, 94 \%$ ). Due to the insolubility of $\left[\mathrm{CpIrCl}_{2}\right]_{x}$ analysis by NMR was precluded. Anal. Calcd for $\left[\mathrm{CpIrCl}_{2}\right]_{x}: \mathrm{C}, 18.30 ; \mathrm{H}, 1.54 ; \mathrm{Cl}$, 21.60. Found: C, $18.73 ; \mathrm{H}, 1.76 ; \mathrm{Cl}, 21.19$.

$\mathrm{CpIrCl}_{2}\left(\mathrm{PPh}_{3}\right)$. [CpIrCl $]_{x}(80 \mathrm{mg})$ and $\mathrm{PPh}_{9}(64 \mathrm{mg}, 2.44 \times$ $\left.10^{-4} \mathrm{~mol}\right)$ in thf $\left(10 \mathrm{~cm}^{3}\right)$ were stirred at room temperature overnight. The suspension changed color from orange to yellow. The thf was removed under vacuum, and the yellow solid was crystallized from $\mathrm{CH}_{2} \mathrm{Cl}_{2}$ /hexane $(134 \mathrm{mg}, 93 \%)$. ${ }^{1} \mathrm{H}$ NMR (80 $\left.\mathrm{MHz} \mathrm{CDCl}_{3}\right): \delta 7.51\left(15 \mathrm{H}, \mathrm{m}, 3 \times \mathrm{C}_{6} \mathrm{H}_{5}\right), 5.38(5 \mathrm{H}, \mathrm{d}, J(\mathrm{HP})$ $=1.5 \mathrm{~Hz}, \mathrm{C}_{5} \mathrm{H}_{5}$ ). MS (peaks are quoted for ${ }^{193} \mathrm{Ir}$ and ${ }^{35} \mathrm{Cl}$ isotopomers): $m / z 590\left(7 \%, \mathrm{M}^{+}\right), 262\left(100 \%, \mathrm{PPh}_{3}\right)$.

$\operatorname{CpIr}\left(\mathrm{CH}_{3}\right)_{2}\left(\mathrm{PPh}_{\mathrm{s}}\right) . \mathrm{CpIrCl}_{2}\left(\mathrm{PPh}_{3}\right)\left(250 \mathrm{mg}, 4.22 \times 10^{-4} \mathrm{~mol}\right)$ was suspended in diethyl ether and cooled to $0^{\circ} \mathrm{C}$. $\mathrm{MeMgBr}$ (diluted to $1 \mathrm{~mol} \mathrm{dm}^{-3}$ ) was slowly added. The yellow solid slowly changed to a yellow resinous material. After overnight stirring, a pale yellow precipitate formed. The solution was filtered and concentrated, and the $\mathrm{CpIr}\left(\mathrm{CH}_{3}\right)_{2}\left(\mathrm{PPh}_{3}\right)$ (pale yellow solid) was extracted with hexane and purified by passing the solution through a Celite column: yield $138 \mathrm{mg}(58 \%) . \operatorname{NMR}\left(\mathrm{C}_{7} \mathrm{D}_{8}\right):{ }^{1} \mathrm{H}$ $\delta 7.27\left(15 \mathrm{H}, \mathrm{m}, 3 \times \mathrm{C}_{6} \mathrm{H}_{5}\right), 4.8\left(5 \mathrm{H}, \mathrm{d}, J(\mathrm{HP})=1.8 \mathrm{~Hz}, \mathrm{C}_{5} \mathrm{H}_{5}\right)$, $0.96\left(6 \mathrm{H}, \mathrm{d}, J(\mathrm{HP})=5.1 \mathrm{~Hz}, 2 \times \mathrm{CH}_{3}\right) ;{ }^{31} \mathrm{P}\left\{{ }^{1} \mathrm{H}\right\} \delta 10.46(\mathrm{~s}) . \mathrm{MS}$ (peaks are quoted for ${ }^{193} \mathrm{Ir}$ isotopomers): $m / z 550\left(18 \%, \mathrm{M}^{+}\right), 519$ (100\%). Accurate mass: calcd 548.1378, found 548.1389.

[CpIr $\left.\left(\mathrm{C}_{2} \mathrm{H}_{4}\right) \mathbf{H}\left(\mathrm{PPh}_{3}\right)\right] \mathrm{PF}_{6} . \operatorname{CpIr}\left(\mathrm{CH}_{3}\right)_{2}\left(\mathrm{PPh}_{3}\right)(130 \mathrm{mg}, 2.36$ $\times 10^{-4} \mathrm{~mol}$ ) was dissolved in $\mathrm{CH}_{2} \mathrm{Cl}_{2}\left(5 \mathrm{~cm}^{3}\right)$. The yellow solution was cooled to $-78^{\circ} \mathrm{C}$, and $\left[\mathrm{Ph}_{3} \mathrm{C}\right] \mathrm{PF}_{6}\left(90 \mathrm{mg}, 2.3 \times 10^{-4} \mathrm{~mol}\right)$ in $\mathrm{CH}_{2} \mathrm{Cl}_{2}\left(2 \mathrm{~cm}^{3}\right)$ was added in portions. The reaction mixture was allowed to warm to room temperature slowly $(>1 \mathrm{~h}) . \mathrm{Et}_{2} \mathrm{O}(2$ $\mathrm{cm}^{3}$ ) was added and the solution cooled to $0{ }^{\circ} \mathrm{C}$. A precipitate was formed which was washed with hexane and thf to give a white solid: yield $85 \mathrm{mg}(67 \%)$. NMR $\left(\mathrm{CD}_{2} \mathrm{Cl}_{2}\right)$ : ${ }^{1} \mathrm{H}(90 \mathrm{MHz})$ $\delta 7.39\left(15 \mathrm{H}, \mathrm{m}, 3 \times \mathrm{C}_{6} \mathrm{H}_{5}\right), 5.72\left(5 \mathrm{H}, \mathrm{d}, J(\mathrm{HP})=1 \mathrm{~Hz}, \mathrm{C}_{5} \mathrm{H}_{5}\right)$, $3.00\left(2 \mathrm{H}, \mathrm{m}, \mathrm{C}_{2} \mathrm{H}_{4}\right), 2.40\left(2 \mathrm{H}, \mathrm{m}, \mathrm{C}_{2} \mathrm{H}_{4}\right),-15.60(1 \mathrm{H}, \mathrm{d}, J(\mathrm{HP})$ $=27 \mathrm{~Hz}, \mathrm{IrH}) ;{ }^{13} \mathrm{C}\left\{{ }^{1} \mathrm{H}\right\} \delta 133.5(\mathrm{~d}, J(\mathrm{CP})=9 \mathrm{~Hz}), 132.5(\mathrm{~s}), 129.5$ $(\mathrm{d}, J(\mathrm{CP})=12 \mathrm{~Hz}), 129.3(\mathrm{~s})\left(3 \times \mathrm{C}_{6} \mathrm{H}_{5}\right), 89.7\left(\mathrm{~s}, \mathrm{C}_{5} \mathrm{H}_{5}\right), 29.3(\mathrm{~s}$, $\mathrm{C}_{2} \mathrm{H}_{4}$ ).

$\operatorname{CpIr}\left(\mathrm{C}_{2} \mathrm{H}_{4}\right)\left(\mathrm{PPh}_{3}\right)$. [CpIr $\left.\left(\mathrm{C}_{2} \mathrm{H}_{4}\right) \mathrm{H}\left(\mathrm{PPh}_{3}\right)\right] \mathrm{PF}_{6}(80 \mathrm{mg}, 1.15$ $\left.\times 10^{-4} \mathrm{~mol}\right)$ was suspended in thf $\left(10 \mathrm{~cm}^{3}\right)$. Excess $\mathrm{NaH}$ suspended in thf was added dropwise with vigorous stirring. The reaction

(12) Haddleton, D. M.; McCamley, A.; Perutz, R. N. J. Am. Chem. Soc. 1988, 110, 1810

(13) Mayer, J. M.; Calabrese J. C. Organometallics 1984, 3, 1292.

(14) Szajek, L. P.; Lawson, R. J.; Shapley, J. R. Organometallics 1991, 10,357 . 
mixture was heated to $35-40^{\circ} \mathrm{C}$. Addition of $\mathrm{NaH}$ was continued until the evolution of gas subsided (ca. $30 \mathrm{~min}$ ). The yellow solution was filtered and pumped to dryness. The solid was extracted with $3 \times 10 \mathrm{~cm}^{3}$ of hexane and filtered. The filtrate was pumped to dryness to give a yellow solid: yield $(45 \mathrm{mg}, 71 \%)$. NMR: ${ }^{1} \mathrm{H}$ (dmso) $\delta 7.45-7.37\left(15 \mathrm{H}, \mathrm{m}, 3 \times \mathrm{C}_{6} \mathrm{H}_{5}\right), 5.05(5 \mathrm{H}$, d, $\left.J(\mathrm{HP})=1.1 \mathrm{~Hz}, \mathrm{C}_{5} \mathrm{H}_{5}\right), 1.84\left(2 \mathrm{H}, \mathrm{m}, \mathrm{C}_{2} \mathrm{H}_{4}\right), 0.196\left(2 \mathrm{H}, \mathrm{m}, \mathrm{C}_{2} \mathrm{H}_{4}\right)$; ${ }^{13} \mathrm{C}\left\{{ }^{1} \mathrm{H}\right\}\left(\mathrm{C}_{7} \mathrm{D}_{8}\right) \delta 138.4,135.6,135.4,128.5\left(3 \times \mathrm{C}_{6} \mathrm{H}_{5}\right), 82.52\left(\mathrm{C}_{5} \mathrm{H}_{5}\right)$, $7.96\left(\mathrm{CH}_{2}=\mathrm{CH}_{2}\right) ;{ }^{31} \mathrm{P}\left\{{ }^{1} \mathrm{H}\right\}\left(90 \mathrm{MHz}, \mathrm{C}_{6} \mathrm{D}_{6}\right) \delta 15.9(\mathrm{~s})$. Accurate mass: calcd 546.120, found 546.1221.

$\mathrm{CpIr}\left(\mathrm{PPh}_{3}\right)\left(\mathrm{C}_{6} \mathrm{H}_{5}\right) \mathrm{Br}$ and $\mathrm{CpIr}\left(\mathrm{PPh}_{3}\right)\left(\mathrm{C}_{2} \mathrm{H}_{3}\right) \mathrm{Br}$. CpIr$\left(\mathrm{C}_{2} \mathrm{H}_{4}\right)_{2}\left(50 \mathrm{mg}, 1.6 \times 10^{-4} \mathrm{~mol}\right)$ was irradiated $(\lambda>365 \mathrm{~nm}, 84$ h) in benzene $\left(1.5 \mathrm{~cm}^{3}\right)$ in the presence of $\mathrm{PPh}_{3}\left(45 \mathrm{mg}, 1.7 \times 10^{-4}\right.$ mol). The products were $\mathrm{CpIr}\left(\mathrm{PPh}_{3}\right)\left(\mathrm{C}_{6} \mathrm{H}_{5}\right) \mathrm{H}, \mathrm{CpIr}\left(\mathrm{PPh}_{3}\right)$ $\left(\mathrm{C}_{2} \mathrm{H}_{3}\right) \mathrm{H}$, and $\mathrm{CpIr}\left(\mathrm{PPh}_{3}\right)\left(\mathrm{C}_{2} \mathrm{H}_{4}\right)$. The solvent was removed and bromination of the hydrides was carried out according to the literature procedure. ${ }^{15}$ The crude mixture was dissolved in thf $\left(10 \mathrm{~cm}^{3}\right)$ and cooled to $-78^{\circ} \mathrm{C} . \mathrm{CHBr}_{3}\left(45 \mathrm{mg}, 1.8 \times 10^{-4} \mathrm{~mol}\right)$ diluted in thf $\left(4 \mathrm{~cm}^{3}\right)$ was added slowly. The mixture was left for $15 \mathrm{~min}$ and then allowed to warm to room temperature, and the solvent was removed. $\mathrm{CpIr}\left(\mathrm{PPh}_{3}\right)\left(\mathrm{C}_{6} \mathrm{H}_{5}\right) \mathrm{Br}$ and $\mathrm{CpIr}\left(\mathrm{PPh}_{3}\right)-$ $\left(\mathrm{C}_{2} \mathrm{H}_{3}\right) \mathrm{Br}$ were separated on a silica column using $60 \%$ toluene/ $40 \%$ hexane as the eluant.

$\mathrm{CpIr}\left(\mathrm{PPh}_{3}\right)\left(\mathrm{C}_{6} \mathrm{H}_{5}\right) \mathrm{Br}:{ }^{1} \mathrm{H}$ NMR $\left(\mathrm{CDCl}_{3}\right) \delta 7.5\left(1 \mathrm{H}, \mathrm{d}, J\left(\mathrm{H}_{0} \mathrm{H}_{\mathrm{m}}\right)\right.$ $=11.5 \mathrm{~Hz}, 0$-phenyl hydrogen), $7.33\left(15 \mathrm{H}, \mathrm{m}, 3 \times \mathrm{C}_{6} \mathrm{H}_{5}\right), 6.81$ $(3 \mathrm{H}, \mathrm{m}, p$ - and $m$-phenyl hydrogens), $5.33(5 \mathrm{H}, \mathrm{d}, J(\mathrm{HP})=2$ $\mathrm{Hz}, \mathrm{C}_{5} \mathrm{H}_{5}$ ), one o-phenyl hydrogen signal is masked by the phosphine phenyl signals; ${ }^{13} \mathrm{C}\left\{{ }^{1} \mathrm{H}\right\} \mathrm{NMR}\left(\mathrm{CDCl}_{3}\right) \delta 144.1$ (d, $J(\mathrm{CP})$ $=2.8 \mathrm{~Hz}), 134.9(\mathrm{~d}, J(\mathrm{CP})=2.8 \mathrm{~Hz}), 128.6(\mathrm{~d}, J(\mathrm{CP})=2.8 \mathrm{~Hz}$, $\left.3 \times \mathrm{C}_{6} \mathrm{H}_{5}\right), 132.7\left(\mathrm{~d}, J(\mathrm{CP})=3 \mathrm{~Hz}, i-\mathrm{C}_{6} \mathrm{H}_{5}\right), 131.0(\mathrm{~d}, J(\mathrm{CP})=2.4$ $\left.\mathrm{Hz}, 0-\mathrm{C}_{6} \mathrm{H}_{5}\right), 127.7\left(\mathrm{~s}, m-\mathrm{C}_{6} \mathrm{H}_{5}\right), 123.1\left(\mathrm{~s}, p-\mathrm{C}_{6} \mathrm{H}_{5}\right), 86.7$ (d, J(CP) $\left.=3.1 \mathrm{~Hz}, \mathrm{C}_{5} \mathrm{H}_{5}\right) ;{ }^{31} \mathrm{P}\left\{{ }^{1} \mathrm{H}\right\} \mathrm{NMR}\left(\mathrm{CDCl}_{3}\right) \delta 0.7 ; \mathrm{MS}$ (peaks are quoted for ${ }^{193} \mathrm{Ir},{ }^{81} \mathrm{Br}$ isotopomers) $\mathrm{m} / z 678\left(5 \%, \mathrm{M}^{+}\right), 262(100 \%$, $\left.\mathrm{PPh}_{3}\right)$. Accurate mass: calcd 678.0486, found 678.0491.

$\mathrm{CpIr}\left(\mathrm{PPh}_{3}\right)\left(\mathrm{C}_{2} \mathrm{H}_{3}\right) \mathrm{Br}$ : ${ }^{1} \mathrm{H} \mathrm{NMR}\left(90 \mathrm{MHz}, \mathrm{CDCl}_{3}\right)$ (the annotation for the vinyl protons is the same as defined for the vinyl hydride complexes in the diagram below $) \delta 7.96\left(1 \mathrm{H}\right.$, ddd, $J\left(\mathrm{H}_{\mathrm{c}} \mathrm{H}_{\mathrm{a}}\right)$ $\left.=17 \mathrm{~Hz}, J\left(\mathrm{H}_{\mathrm{c}} \mathrm{H}_{\mathrm{b}}\right)=10 \mathrm{~Hz}, J\left(\mathrm{H}_{\mathrm{c}} \mathrm{P}\right)=4.2 \mathrm{~Hz}, \mathrm{H}_{\mathrm{c}}\right), 7.5(15 \mathrm{H}, \mathrm{m}$, $\left.3 \times \mathrm{C}_{6} \mathrm{H}_{5}\right), 6.06\left(1 \mathrm{H}\right.$, ddd, $J\left(\mathrm{H}_{\mathrm{b}} \mathrm{H}_{\mathrm{c}}\right)=10 \mathrm{~Hz}, J\left(\mathrm{H}_{\mathrm{b}} \mathrm{P}\right)=3.2 \mathrm{~Hz}$ $\left.J\left(\mathrm{H}_{\mathrm{b}} \mathrm{H}_{\mathrm{a}}\right)=1.8 \mathrm{~Hz}, \mathrm{H}_{\mathrm{b}}\right), 5.28\left(1 \mathrm{H}, \mathrm{ddd}, J\left(\mathrm{H}_{\mathrm{a}} \mathrm{H}_{\mathrm{c}}\right)=17 \mathrm{~Hz}, J\left(\mathrm{H}_{\mathrm{a}} \mathrm{H}_{\mathrm{b}}\right)\right.$ $\left.=1.8 \mathrm{~Hz}, J(\mathrm{HP})=1.8 \mathrm{~Hz}, \mathrm{H}_{\mathrm{a}}\right), 5.14(5 \mathrm{H}, \mathrm{d}, J(\mathrm{HP})=1.5 \mathrm{~Hz}$, $\left.\mathrm{C}_{5} \mathrm{H}_{5}\right) ;{ }^{13} \mathrm{C}\left\{{ }^{1} \mathrm{H}\right\} \mathrm{NMR}\left(\mathrm{CDCl}_{3}\right) \delta 134.9(\mathrm{~d}, J(\mathrm{CP})=10 \mathrm{~Hz}), 131.7$ $(\mathrm{d}, J(\mathrm{CP})=3 \mathrm{~Hz}), 128.7\left(\mathrm{~d}, J(\mathrm{CP})=11 \mathrm{~Hz}, \mathrm{P}\left(\mathrm{C}_{6} \mathrm{H}_{5}\right)_{3}\right), 127.2(\mathrm{~d}$, $\left.J(\mathrm{CP})=15 \mathrm{~Hz}, \mathrm{CH}=\mathrm{CH}_{2}\right), 124.7\left(\mathrm{~d}, J(\mathrm{CP})=4 \mathrm{~Hz}, \mathrm{CH}=\mathrm{CH}_{2}\right.$ ), $86.9\left(\mathrm{~d}, J(\mathrm{CP})=3 \mathrm{~Hz}, \mathrm{C}_{5} \mathrm{H}_{5}\right) ;{ }^{31} \mathrm{P}\left\{{ }^{1} \mathrm{H}\right\} \mathrm{NMR}\left(\mathrm{CDCl}_{3}\right) \delta 1.0(\mathrm{~s}) ; \mathrm{MS}$ (peaks are quoted for ${ }^{193} \mathrm{Ir},{ }^{81} \mathrm{Br}$ isotopomers) $\mathrm{m} / z 628\left(6 \%, \mathrm{M}^{+}\right.$), $262\left(100 \%, \mathrm{PPh}_{3}\right)$.

NMR data for $\mathrm{CpIr}(\mathrm{L})(\mathrm{R}) \mathrm{H}\left(\mathrm{L}=\mathrm{CO}, \mathrm{PPh}_{3}, \mathrm{R}=\right.$ vinyl, alkyl, aryl) are as follows. The vinyl protons are assigned according to

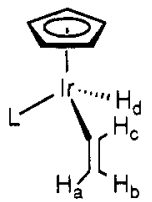

NMR Data for Iridium Hydride Complexes. CpIr(CO)$\left(\mathrm{C}_{2} \mathrm{H}_{3}\right)$ H. NMR: ${ }^{1} \mathrm{H}\left(\mathrm{C}_{6} \mathrm{D}_{5} \mathrm{CD}_{3}\right) \delta 7.51\left(1 \mathrm{H}\right.$, dd, $J\left(\mathrm{H}_{\mathrm{c}} \mathrm{H}_{\mathrm{a}}\right)=17.2$ $\left.\mathrm{Hz}, J\left(\mathrm{H}_{\mathrm{c}} \mathrm{H}_{\mathrm{b}}\right)=9.8 \mathrm{~Hz}, \mathrm{H}_{\mathrm{c}}\right), 6.49\left(1 \mathrm{H}, \mathrm{dd}, J\left(\mathrm{H}_{\mathrm{b}} \mathrm{H}_{\mathrm{c}}\right)=9.8 \mathrm{~Hz}\right.$, $\left.J\left(\mathrm{H}_{\mathrm{b}} \mathrm{H}_{\mathrm{a}}\right)=2.3 \mathrm{~Hz}, \mathrm{H}_{\mathrm{b}}\right), 5.74\left(1 \mathrm{H}, \mathrm{dd}, J\left(\mathrm{H}_{\mathrm{a}} \mathrm{H}_{\mathrm{c}}\right)=17.2 \mathrm{~Hz}, J\left(\mathrm{H}_{\mathrm{a}} \mathrm{H}_{\mathrm{b}}\right)\right.$ $\left.=2.3 \mathrm{~Hz}, \mathrm{H}_{\mathrm{c}}\right), 4.77\left(5 \mathrm{H}, \mathrm{s}, \mathrm{C}_{5} \mathrm{H}_{5}\right),-15.57(1 \mathrm{H}, \mathrm{s}, \mathrm{IrH}) ;{ }^{13} \mathrm{C}\left\{{ }^{1} \mathrm{H}\right\}$ $\left(\mathrm{C}_{6} \mathrm{D}_{5} \mathrm{CD}_{3}\right) \delta 170.4(\mathrm{CO}), 128.4\left(\mathrm{CH}_{2}\right), 103.4(\mathrm{CH}), 87.7\left(\mathrm{C}_{5} \mathrm{H}_{5}\right)$.

$\operatorname{CpIr}(\mathrm{CO})\left(\mathrm{C}_{6} \mathrm{D}_{5}\right) \mathrm{H} .{ }^{1} \mathrm{H}$ NMR $\left(\mathrm{C}_{6} \mathrm{D}_{6}\right): \delta 4.72\left(5 \mathrm{H}, \mathrm{s}, \mathrm{C}_{5} \mathrm{H}_{5}\right)$, $-15.34(1 \mathrm{H}, \mathrm{s}, \mathrm{IrH})$.

$\operatorname{CpIr}(\mathrm{CO})\left(\mathrm{C}_{6} \mathrm{D}_{11}\right) \mathrm{H} .{ }^{1} \mathrm{H}$ NMR $\left(\mathrm{C}_{6} \mathrm{D}_{12}\right): \delta 5.26\left(5 \mathrm{H}, \mathrm{s}, \mathrm{C}_{5} \mathrm{H}_{5}\right)$, $-16.64(1 \mathrm{H}, \mathrm{s}, \mathrm{IrH})$.

$\operatorname{CpIr}\left(\mathrm{PPh}_{3}\right)\left(\mathrm{C}_{2} \mathrm{H}_{3}\right) \mathrm{H}$. NMR: ${ }^{1} \mathrm{H}\left(\mathrm{C}_{6} \mathrm{D}_{6}\right) \delta 7.85(1 \mathrm{H}$, dddd, $J\left(\mathrm{H}_{\mathrm{c}} \mathrm{H}_{\mathrm{a}}\right)=17 \mathrm{~Hz}, J\left(\mathrm{H}_{\mathrm{c}} \mathrm{H}_{\mathrm{b}}\right)=10 \mathrm{~Hz}, J\left(\mathrm{H}_{\mathrm{c}} \mathrm{P}\right)=7 \mathrm{~Hz}, J\left(\mathrm{H}_{\mathrm{c}} \mathrm{H}_{\mathrm{d}}\right)=$ $\left.1 \mathrm{~Hz}, \mathrm{H}_{\mathrm{c}}\right), 7.6-7.0\left(15 \mathrm{H}, \mathrm{m}, 3 \times \mathrm{C}_{6} \mathrm{H}_{5}\right), 6.58\left(1 \mathrm{H}\right.$, ddd, $J\left(\mathrm{H}_{\mathrm{b}} \mathrm{H}_{\mathrm{a}}\right)$ $\left.=3 \mathrm{~Hz}, J\left(\mathrm{H}_{\mathrm{b}} \mathrm{H}_{\mathrm{c}}\right)=10 \mathrm{~Hz}, J\left(\mathrm{H}_{\mathrm{b}} \mathrm{P}\right)=3 \mathrm{~Hz}, \mathrm{H}_{\mathrm{b}}\right), 5.79(1 \mathrm{H}$, ddd,

(15) Jones, W. D.; Feher, F. J. J. Am. Chem. Soc. 1984, 106, 1650.
$\left.J\left(\mathrm{H}_{\mathrm{a}} \mathrm{P}\right)=1.5 \mathrm{~Hz}, J\left(\mathrm{H}_{\mathrm{a}} \mathrm{H}_{\mathrm{b}}\right)=3 \mathrm{~Hz}, J\left(\mathrm{H}_{\mathrm{a}} \mathrm{H}_{\mathrm{c}}\right)=17 \mathrm{~Hz}, \mathrm{H}_{\mathrm{a}}\right), 4.95$ $\left(5 \mathrm{H}, \mathrm{d}, J(\mathrm{HP})=1 \mathrm{~Hz}, \mathrm{C}_{5} \mathrm{H}_{5}\right),-16.05(1 \mathrm{H}, \mathrm{d}, J(\mathrm{HP})=33.1 \mathrm{~Hz}$, IrH); ${ }^{11} \mathrm{P}\left\{{ }^{1} \mathrm{H}\right\}\left(\mathrm{C}_{6} \mathrm{D}_{6}\right) \delta 17.32(\mathrm{~s})$.

$\operatorname{CpIr}\left(\mathbf{P P h}_{3}\right)\left(\mathbf{C}_{6} \mathrm{H}_{5}\right)$ H. NMR: ${ }^{1} \mathrm{H}\left(\mathrm{C}_{6} \mathrm{D}_{6}\right) \delta 7.43-7.0(15 \mathrm{H}, \mathrm{m}$, $\left.3 \times \mathrm{C}_{6} \mathrm{H}_{5}\right), 6.8(3 \mathrm{H}, \mathrm{t}, m$ - and $p$-phenyl hydrogens), $4.94(5 \mathrm{H}$, d, $\left.J(\mathrm{HP})=1.3 \mathrm{~Hz}, \mathrm{C}_{5} \mathrm{H}_{5}\right),-16.28(1 \mathrm{H}, \mathrm{d}, J(\mathrm{HP})=35.5 \mathrm{~Hz}, \mathrm{IrH})$, $a$-phenyl hydrogen signal masked by the phosphine phenyl signals; ${ }^{31} \mathrm{P}\left\{{ }^{1} \mathrm{H}\right\}\left(\mathrm{C}_{6} \mathrm{D}_{6}\right) \delta 15.46(\mathrm{~s})$.

\section{Results}

(a) $\mathrm{CpIr}\left(\mathrm{PPh}_{\mathbf{3}}\right)\left(\mathrm{C}_{2} \mathrm{H}_{4}\right) . \mathrm{CpIr}\left(\mathrm{PPh}_{3}\right)\left(\mathrm{C}_{2} \mathrm{H}_{4}\right)$ was synthesized via hydride abstraction from $\mathrm{CpIr}\left(\mathrm{CH}_{3}\right)_{2}\left(\mathrm{PPh}_{3}\right)$ with triphenylcarbenium tetrafluoroborate, as described previously for $\mathrm{CpIr}\left(\mathrm{PMe}_{3}\right)\left(\mathrm{C}_{2} \mathrm{H}_{4}\right)$ and related compounds.., 16

(i) Photoproduct Studies in Solution. Photolysis of $\mathrm{CpIr}\left(\mathrm{PPh}_{3}\right)\left(\mathrm{C}_{2} \mathrm{H}_{4}\right)$ has the potential of causing three different $\mathrm{C}-\mathrm{H}$ activation processes: (i) insertion into the ethene ligand to form $\mathrm{CpIr}\left(\mathrm{PPh}_{3}\right)\left(\mathrm{C}_{2} \mathrm{H}_{3}\right) \mathrm{H}$, (ii) expulsion of ethene and cyclometalation of the triphenylphosphine to form $\mathrm{CpIr}\left(\mathrm{PPh}_{2} \mathrm{C}_{6} \mathrm{H}_{4}\right) \mathrm{H}$, (iii) expulsion of the ethene and insertion into the $\mathrm{C}-\mathrm{H}$ bonds of the solvent, $\mathrm{XH}$, yielding $\mathrm{CpIr}\left(\mathrm{PPh}_{3}\right)(\mathrm{X}) \mathrm{H}$. Irradiation of $\mathrm{CpIr}\left(\mathrm{PPh}_{3}\right)$ $\left(\mathrm{C}_{2} \mathrm{H}_{4}\right)$ in cold toluene solution $(198 \mathrm{~K}, \lambda>295 \mathrm{~nm})$ resulted in conversion to a single product readily identified from the NMR spectra as $\mathrm{CpIr}\left(\mathrm{PPh}_{3}\right)\left(\mathrm{C}_{2} \mathrm{H}_{3}\right)(\mathrm{H})$. A $66 \%$ conversion was achieved after $5 \mathrm{~h}$ of irradiation. When photolysis of $\mathrm{CpIr}\left(\mathrm{PPh}_{3}\right)\left(\mathrm{C}_{2} \mathrm{H}_{4}\right)$ was carried out in benzene at room temperature $(60 \mathrm{~min}, \lambda>290 \mathrm{~nm})$, three products were formed in the ratio $14: 2: 1$, of which the major product was the vinyl hydride complex, the product formed in intermediate yield was identified as $\mathrm{CpIr}\left(\mathrm{PPh}_{3}\right)(\mathrm{Ph}) \mathrm{H}$, and the minor product remains identified only as a cyclopentadienyliridium hydride. When $\mathrm{Cp} \operatorname{Ir}\left(\mathrm{PPh}_{3}\right)$ $\left(\mathrm{C}_{2} \mathrm{H}_{4}\right)$ was irradiated in $\mathrm{C}_{6} \mathrm{D}_{6}$, analogous products were obtained, but the additional complication of exchange reactions was signaled by the formation of $\mathrm{CpIr}\left(\mathrm{PPh}_{3}\right)$ $\left(\mathrm{C}_{6} \mathrm{D}_{5}\right) \mathrm{H}$ rather than $\mathrm{CpIr}\left(\mathrm{PPh}_{3}\right)\left(\mathrm{C}_{6} \mathrm{D}_{5}\right) \mathrm{D}$. Identification of the $\mathrm{C}-\mathrm{H}$ activation products was confirmed by conversion to the air-stable complexes $\operatorname{CpIr}\left(\mathrm{PPh}_{3}\right)\left(\mathrm{C}_{6} \mathrm{D}_{5}\right) \mathrm{Br}$ and $\mathrm{CpIr}\left(\mathrm{PPh}_{3}\right)\left(\mathrm{C}_{2} \mathrm{H}_{3}\right) \mathrm{Br}$ by reaction with $\mathrm{CHBr}_{3}$.

(ii) Thermal Stability of $\operatorname{CpIr}\left(\mathrm{PPh}_{3}\right)\left(\mathrm{C}_{2} \mathrm{H}_{3}\right) \mathrm{H}$. A sample of $\mathrm{CpIr}\left(\mathrm{PPh}_{3}\right)\left(\mathrm{C}_{2} \mathrm{H}_{3}\right) \mathrm{H}$ in dmso-d $d_{6}$ was heated at $391 \pm 2 \mathrm{~K}$. The thermal isomerization of $\mathrm{CpIr}\left(\mathrm{PPh}_{3}\right)$ $\left(\mathrm{C}_{2} \mathrm{H}_{3}\right) \mathrm{H}$ to $\mathrm{CpIr}\left(\mathrm{PPh}_{3}\right)\left(\mathrm{C}_{2} \mathrm{H}_{4}\right)$ was monitored as a function of time by ${ }^{1} \mathrm{H}$ NMR. In each spectrum the hydride signal was normalized to the residual proton signal of the dmso. We did not use the integration of the $C p$ resonance because the very long relaxation time (typically $30 \mathrm{~s}$ ) makes it necessary to use extraordinarily long delays between pulses in order to obtain accurate peak integrations. ${ }^{17}$ Typically, the relaxation times of metal hydrides are less than $1 \mathrm{~s} .{ }^{18}$ The rate constant and $\Delta G^{*}$ for the isomerization were determined as $(7.0 \pm 1.4) \times 10^{-6} \mathrm{~s}^{-1}$ and $135 \pm 3 \mathrm{~kJ} \mathrm{~mol}^{-1}$, respectively, at this temperature.

(b) $\mathrm{CpIr}(\mathrm{CO})\left(\mathrm{C}_{2} \mathrm{H}_{4}\right)$. (i) Photoproduct Studies in Cryogenic Solid Matrices. A sample of $\mathrm{CpIr}(\mathrm{CO})\left(\mathrm{C}_{2} \mathrm{H}_{4}\right)$ in solid toluene was irradiated at $77 \mathrm{~K}(7 \mathrm{~h}, \lambda>290 \mathrm{~nm})$ and subsequently thawed. Room temperature ${ }^{1} \mathrm{H}$ and ${ }^{13} \mathrm{C}$ NMR spectra demonstrated $60 \%$ conversion to the oxidative addition product $\mathrm{CpIr}(\mathrm{CO})\left(\mathrm{C}_{2} \mathrm{H}_{3}\right) \mathrm{H}$.

(16) Werner, H.; Feser, R. J. Organomet. Chem. 1982, 232, 351.

(17) Partridge, M. G. D. Phil. thesis, University of York, 1992.

(18) Hamilton, D. G.; Crabtree, R. H. J. Am. Chem. Soc. 1988, 110 , 
Table I. Principal IR Bands $\left(\mathrm{cm}^{-1}\right)$ of the Photoproducts of $\mathrm{CpIr}(\mathrm{CO})\left(\mathrm{C}_{2} \mathrm{H}_{4}\right)$ in Solid Matrices

\begin{tabular}{|c|c|c|c|c|c|}
\hline \multicolumn{4}{|c|}{ matrix type } & \multirow{2}{*}{\multicolumn{2}{|c|}{ assignment }} \\
\hline Ar & $\mathrm{CH}_{4}$ & $\begin{array}{l}2 \% \mathrm{CO} \\
98 \% \mathrm{Ar}\end{array}$ & $\mathrm{N}_{2}$ & & \\
\hline \multirow{9}{*}{$\begin{array}{r}2028 \\
2166 \\
1577 \\
1375 \\
1259 \\
994\end{array}$} & 2015 & 2020 & 2022 & $\nu(\mathrm{CO})$ & $\mathrm{CpIr}(\mathrm{CO})\left(\mathrm{C}_{2} \mathrm{H}_{3}\right) \mathrm{H}$ \\
\hline & 2193 & & 2199 & $\nu(\mathrm{Ir}-\mathrm{H})$ & \\
\hline & 1570 & 1574 & 1576 & $\nu\left(\mathrm{CH}=\mathrm{CH}_{2}\right)$ & \\
\hline & & 1379 & 1385 & $\delta\left(\mathrm{CH}_{2}\right)$ & \\
\hline & & 1259 & 1263 & $\delta(\mathrm{CH})$ & \\
\hline & 995 & 995 & 996 & $\rho(\mathrm{CH})$ & \\
\hline & $\begin{array}{l}2005 \\
2193\end{array}$ & & & $\begin{array}{l}\nu(\mathrm{CO}) \\
\nu(\mathrm{Ir}-\mathrm{H})\end{array}$ & $\mathrm{CpIr}(\mathrm{CO})\left(\mathrm{CH}_{3}\right) \mathrm{H}$ \\
\hline & & 2040 & & $\nu(\mathrm{CO})_{\mathrm{sym}}$ & $\mathrm{CpIr}(\mathrm{CO})_{2}$ \\
\hline & & 1972 & 2162 & $\begin{array}{l}\nu(\mathrm{CO})_{\text {asym }} \\
\nu\left(\mathrm{N}_{2}\right)\end{array}$ & $\operatorname{CpIr}(\mathrm{CO})\left(\mathrm{N}_{2}\right)$ \\
\hline
\end{tabular}

The products from the irradiation of $\mathrm{CpIr}(\mathrm{CO})\left(\mathrm{C}_{2} \mathrm{H}_{4}\right)$ in low temperature gas matrices are summarized in Table I. When $\mathrm{CpIr}(\mathrm{CO})\left(\mathrm{C}_{2} \mathrm{H}_{4}\right)$ was deposited in an argon matrix at $20 \mathrm{~K}$, it exhibited a CO-stretching band at $1987 \mathrm{~cm}^{-1}$. The electronic absorption spectrum showed a band at 243 $\mathrm{nm}$ with a shoulder at $265 \mathrm{~nm}$. After irradiation at $12 \mathrm{~K}$ $(1 \mathrm{~h}, \lambda>230 \mathrm{~nm}$ and $1 \mathrm{~h}, \lambda>290 \mathrm{~nm})$ there was a ca. $64 \%$ depletion of the band at $1987 \mathrm{~cm}^{-1}$. Product bands were observed which were assigned to $\mathrm{CpIr}(\mathrm{CO})\left(\mathrm{C}_{2} \mathrm{H}_{3}\right) \mathrm{H}$ as follows: $2166 \mathrm{~cm}^{-1} \nu(\mathrm{Ir}-\mathrm{H}), 2028 \mathrm{~cm}^{-1} \nu(\mathrm{CO})$ (Figure 1a). In a further experiment a more highly concentrated matrix was irradiated $(12 \mathrm{~K}, 2 \mathrm{~h}, \lambda>290 \mathrm{~nm})$. This enabled the lower intensity IR bands of $\operatorname{CpIr}(\mathrm{CO})\left(\mathrm{C}_{2} \mathrm{H}_{3}\right) \mathrm{H}$ to be observed at $1577 \mathrm{~cm}^{-1} \nu(\mathrm{C}=\mathrm{C}$, vinyl $), 1375 \mathrm{~cm}^{-1} \delta\left(\mathrm{CH}_{2}\right)$, $1259 \mathrm{~cm}^{-1} \delta(\mathrm{CH})$, and $995 \mathrm{~cm}^{-1} \rho(\mathrm{CH})$. These vinyl bands correspond closely to bands of other vinyl hydrides formed in matrices. ${ }^{5}$

Upon deposition into a methane matrix at $20 \mathrm{~K}$ the $\mathrm{CpIr}(\mathrm{CO})\left(\mathrm{C}_{2} \mathrm{H}_{4}\right)$ complex exhibited a $\nu(\mathrm{CO})$ band at 1973 $\mathrm{cm}^{-1}$. UV photolysis at $12 \mathrm{~K}$ resulted in both intra- and intermolecular $\mathrm{C}-\mathrm{H}$ activation. Bands at $2015 \mathrm{~cm}^{-1} \nu$ (CO), $1569 \mathrm{~cm}^{-1} \nu\left(\mathrm{C}=\mathrm{C}\right.$, vinyl), and $995 \mathrm{~cm}^{-1} \rho(\mathrm{CH})$ were assigned to $\mathrm{CpIr}(\mathrm{CO})\left(\mathrm{C}_{2} \mathrm{H}_{3}\right) \mathrm{H}$, while the $\mathrm{CO}$ stretch of the methyl hydride was observed at $2005 \mathrm{~cm}^{-1}$. A band at $2193 \mathrm{~cm}^{-1}$ was assigned to the $\mathrm{Ir}-\mathrm{H}$ stretching modes of both $\mathrm{CpIr}(\mathrm{CO})\left(\mathrm{C}_{2} \mathrm{H}_{3}\right) \mathrm{H}$ and $\mathrm{CpIr}(\mathrm{CO})\left(\mathrm{CH}_{3}\right) \mathrm{H}$. The ratio of the products shows a pronounced wavelength dependence. Irradiation at $\lambda>320 \mathrm{~nm}$ for $30 \mathrm{~min}$ resulted in a $30 \%$ depletion of the starting material and formation of $\mathrm{CpIr}(\mathrm{CO})\left(\mathrm{C}_{2} \mathrm{H}_{3}\right) \mathrm{H}$ and $\mathrm{CpIr}(\mathrm{CO})\left(\mathrm{CH}_{3}\right) \mathrm{H}$ in a ratio of 5.7:1 (based on the absorbances of $\nu(\mathrm{CO})$ bands). However, further photolysis of the matrix at $\lambda>290 \mathrm{~nm}$ for $1 \mathrm{~h}$ resulted in $90 \%$ conversion of the starting material with $\mathrm{CpIr}(\mathrm{CO})\left(\mathrm{C}_{2} \mathrm{H}_{3}\right) \mathrm{H}$ and $\mathrm{CpIr}(\mathrm{CO})\left(\mathrm{CH}_{3}\right) \mathrm{H}$ formed in a ratio of 1.7:1 (Figure 1b). In a separate experiment irradiation of $\mathrm{CpIr}(\mathrm{CO})\left(\mathrm{C}_{2} \mathrm{H}_{4}\right)$ in a methane matrix at $\lambda>365 \mathrm{~nm}$ for $21 \mathrm{~h}$ effected $95 \%$ conversion to $\mathrm{CpIr}(\mathrm{CO})\left(\mathrm{C}_{2} \mathrm{H}_{3}\right) \mathrm{H}$ with almost no methane activation. Further photolysis was then carried out with shorter wavelengths of light. Irradiation with $\lambda>316 \mathrm{~nm}(25 \mathrm{~min})$ resulted in no change in the IR spectrum, whereas the methyl hydride was formed with $\lambda>290 \mathrm{~nm}(2 \mathrm{~h})$. The yield of this product indicated that it could not have originated solely from the remaining $\mathrm{CpIr}(\mathrm{CO})\left(\mathrm{C}_{2} \mathrm{H}_{4}\right)$.

$\mathrm{CpIr}(\mathrm{CO})\left(\mathrm{C}_{2} \mathrm{H}_{4}\right)$ exhibited a band at $1974 \mathrm{~cm}^{-1}$ when it was deposited in a $98 \% \mathrm{Ar} / 2 \% \mathrm{CO}$ matrix at $20 \mathrm{~K}$. Irradiation ( $\lambda>320 \mathrm{~nm}, 60 \mathrm{~min})$ effected $40 \%$ depletion of the starting material and conversion to $\mathrm{CpIr}(\mathrm{CO})$ -

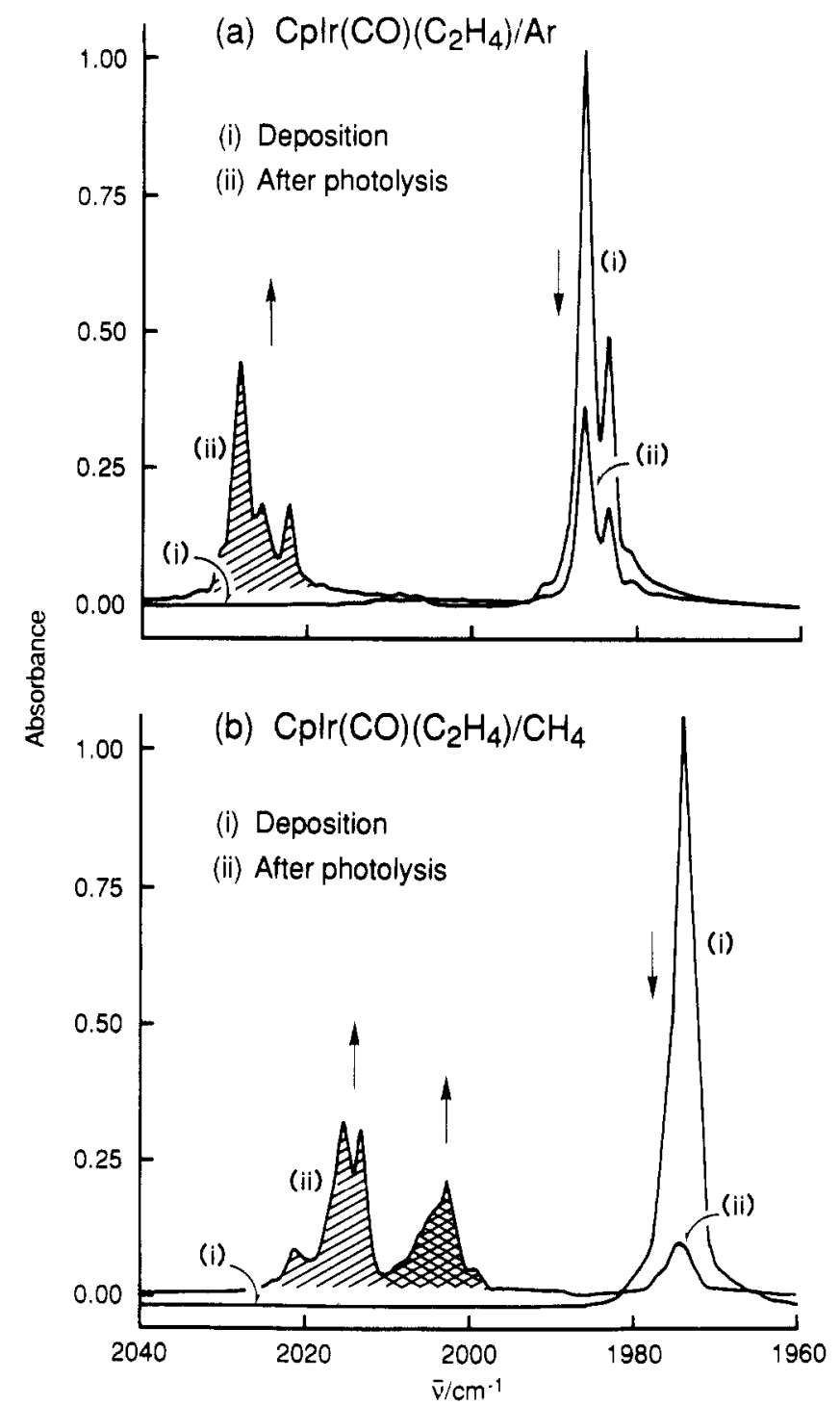

Figure 1. (a) IR spectrum showing (i) the spectrum of $\mathrm{CpIr}$ (CO) $\left(\mathrm{C}_{2} \mathrm{H}_{4}\right)$ in an argon matrix at $12 \mathrm{~K}$ and (ii) the spectrum after $1 \mathrm{~h}, \lambda>320 \mathrm{~nm}$ and $1 \mathrm{~h}, \lambda>290 \mathrm{~nm}$ showing the formation of $\mathrm{CpIr}(\mathrm{CO})\left(\mathrm{C}_{2} \mathrm{H}_{3}\right) \mathrm{H}$. (b) (i) IR spectrum of $\mathrm{CpIr}$ (CO) $\left(\mathrm{C}_{2} \mathrm{H}_{4}\right)$ in a methane matrix at $12 \mathrm{~K}$ and (ii) the spectrum after $30 \mathrm{~min}, \lambda>320 \mathrm{~nm}$ and $1 \mathrm{~h}, \lambda>290 \mathrm{~nm}$ showing the formation of $\operatorname{CpIr}(\mathrm{CO})\left(\mathrm{C}_{2} \mathrm{H}_{3}\right) \mathrm{H}$ and $\operatorname{CpIr}(\mathrm{CO})\left(\mathrm{CH}_{3}\right) \mathrm{H}$.

$\left(\mathrm{C}_{2} \mathrm{H}_{3}\right) \mathrm{H}$ and $\mathrm{CpIr}(\mathrm{CO})_{2}$ (Table $\mathrm{I}$ ) in a ratio of $5: 1 .{ }^{19}$ Further photolysis of the matrix at $\lambda>290 \mathrm{~nm}$ for $60 \mathrm{~min}$ resulted in $60 \%$ conversion of $\mathrm{CpIr}(\mathrm{CO})\left(\mathrm{C}_{2} \mathrm{H}_{4}\right)$ to products in the ratio of $\left[\mathrm{CpIr}(\mathrm{CO})\left(\mathrm{C}_{2} \mathrm{H}_{3}\right) \mathrm{H}\right]:\left[\mathrm{CpIr}(\mathrm{CO})_{2}\right]=1.5: 1$. Again, the product ratios are based on the $\nu(\mathrm{CO})$ band absorbances. No evidence was observed for the existence of any cyclopentadienyl "ring slip" species.

The complex was deposited in a nitrogen matrix at 20 $\mathrm{K}$ in order to determine the extent of $\mathrm{CO}$ loss from CpIr(CO) $\left(\mathrm{C}_{2} \mathrm{H}_{4}\right)$ upon photolysis in a matrix. Irradiation at $\lambda$ $>320 \mathrm{~nm}$ for $1 \mathrm{~h}$ followed by irradiation at $\lambda>295 \mathrm{~nm}$ for $2 \mathrm{~h}$ resulted in formation of only a trace of $\mathrm{CpIr}\left(\mathrm{C}_{2} \mathrm{H}_{4}\right)$ $\left(\mathrm{N}_{2}\right)$.

(ii) Photoproduct Studies in Solution. Photolysis of $\mathrm{CpIr}(\mathrm{CO})\left(\mathrm{C}_{2} \mathrm{H}_{4}\right)$ in benzene- $d_{6}(300 \mathrm{~K}, 1 \mathrm{~h}, \lambda>320 \mathrm{~nm})$ resulted in the formation of both $\operatorname{CpIr}(\mathrm{CO})\left(\mathrm{C}_{2} \mathrm{H}_{3}\right) \mathrm{H}$ and $\mathrm{Cp} \operatorname{Ir}(\mathrm{CO})\left(\mathrm{C}_{6} \mathrm{D}_{5}\right) \mathrm{H}$ with ca. $60 \%$ conversion. $\mathrm{CpIr}(\mathrm{CO})$ -

(19) The $\nu(\mathrm{CO})$ bands of $\mathrm{CpIr}(\mathrm{CO})_{2}$ are assigned according to: Rest, A. J.; Whitwell, I.; Graham, W. A. G.; Hoyano, J. K.; McMaster, A. D. J. Chem. Soc., Chem. Commun. 1984, 624 . 
$\left(\mathrm{C}_{6} \mathrm{D}_{5}\right) \mathrm{H}$ was observed instead of the expected $\mathrm{CpIr}(\mathrm{CO})$ $\left(\mathrm{C}_{6} \mathrm{D}_{5}\right) \mathrm{D}$ due to a rapid $\mathrm{H} / \mathrm{D}$ exchange which resulted in complete conversion to the hydride. As in the matrix isolation studies reported above, the ratio of intra- to intermolecular $\mathrm{C}-\mathrm{H}$ activation exhibited a distinct wavelength dependence. When the sample was irradiated at $\lambda>320 \mathrm{~nm}$, the products were formed in a 1:1 ratio, whereas intermolecular $\mathrm{C}-\mathrm{H}$ activation was favored $([\mathrm{Cp} \operatorname{Ir}(\mathrm{CO})$ $\left.\left.\left(\mathrm{C}_{6} \mathrm{D}_{5}\right) \mathrm{H}\right]:\left[\mathrm{CpIr}(\mathrm{CO})\left(\mathrm{C}_{2} \mathrm{H}_{3}\right) \mathrm{H}\right]=4: 1\right)$ when the sample was irradiated with $\lambda>290 \mathrm{~nm}(300 \mathrm{~K}, 1 \mathrm{~h})$. Irradiation of $\mathrm{CpIr}(\mathrm{CO})\left(\mathrm{C}_{2} \mathrm{H}_{4}\right)$ in cyclohexane- $d_{12}(300 \mathrm{~K}, 1 \mathrm{~h}, \lambda>320$ $\mathrm{nm}$ ) resulted in the formation of $\mathrm{CpIr}(\mathrm{CO})\left(\mathrm{C}_{2} \mathrm{H}_{3}\right) \mathrm{H}$ and $\mathrm{CpIr}(\mathrm{CO})\left(\mathrm{C}_{6} \mathrm{D}_{11}\right) \mathrm{H}$ in a $1: 1$ ratio.

Preliminary time-resolved IR experiments (carried out at Nottingham) on $\mathrm{CpIr}(\mathrm{CO})\left(\mathrm{C}_{2} \mathrm{H}_{4}\right)$ in heptane with 308$\mathrm{nm}$ pulsed excitation show that the heptyl hydride is formed within the response time of the setup $(1 \mu \mathrm{s})$. This indicates that intermolecular $\mathrm{C}-\mathrm{H}$ bond activation arises directly from photolysis of $\mathrm{CpIr}(\mathrm{CO})\left(\mathrm{C}_{2} \mathrm{H}_{4}\right)$. However, it was necessary to investigate if secondary photolysis of $\mathrm{CpIr}(\mathrm{CO})\left(\mathrm{C}_{2} \mathrm{H}_{3}\right) \mathrm{H}$ was also a route to the formation of intermolecular $\mathrm{C}-\mathrm{H}$ bond activation in solution. Therefore, the relative rates of formation of both vinyl and phenyl hydride upon photolysis of $\operatorname{CpIr}(\mathrm{CO})\left(\mathrm{C}_{2} \mathrm{H}_{4}\right)$ in benzene$d_{6}$ were examined. The sample was irradiated with $\lambda>$ $290 \mathrm{~nm}$ at $10^{\circ} \mathrm{C}$, and ${ }^{1} \mathrm{H}$ NMR spectra were recorded at 10-min intervals over a 1-h photolysis time. The ratio of the height of the hydride signal due to $\operatorname{CpIr}(\mathrm{CO})\left(\mathrm{C}_{2} \mathrm{H}_{3}\right) \mathrm{H}$ to that of $\mathrm{CpIr}(\mathrm{CO})\left(\mathrm{C}_{6} \mathrm{D}_{5}\right) \mathrm{H}$ remained constant with time within experimental error. In a further experiment $\mathrm{CpIr}$ $(\mathrm{CO})\left(\mathrm{C}_{2} \mathrm{H}_{4}\right)$ was photolyzed in frozen toluene- $d_{8}(77 \mathrm{~K}, \lambda$ $>290 \mathrm{~nm}, 48 \mathrm{~h}$ ), effecting $80 \%$ conversion to the vinyl hydride. The solution was then irradiated at $283 \mathrm{~K}(\lambda>$ $290 \mathrm{~nm}, 1 \mathrm{~h}$ ). ${ }^{1} \mathrm{H}$ NMR showed that the vinyl hydride was depleted by $50 \%$ with $25 \%$ conversion to the tolyl hydrides. Thus, secondary photolysis of $\operatorname{CpIr}(\mathrm{CO})\left(\mathrm{C}_{2} \mathrm{H}_{3}\right) \mathrm{H}$ is significant, but only when it is the dominant species.

(iii) Determination of the Effect of Solvent Viscosity and Temperature on the Product Ratios. According to the in-cage mechanism of isomerization, the viscosity of the solvent should influence the proportion of intra- to intermolecular activation. Therefore, we investigated the photolysis of $\mathrm{CpIr}(\mathrm{CO})\left(\mathrm{C}_{2} \mathrm{H}_{4}\right)$ in toluene- $d_{8}$ at three temperatures, 188,213 , and $273 \mathrm{~K}$ ( $1 \mathrm{~h}$ at $\lambda>290$ $\mathrm{nm}$ ), the lowest being only $11 \mathrm{deg}$ above the freezing point. Four hydride signals were observed in the ${ }^{1} \mathrm{H}$ NMR spectra at $\delta-15.40,-15.49,-15.57$, and -16.19 . Previous experiments in toluene- $d_{B}$ showed that the high-field signal of the vinyl hydride occurs at $-15.57 \mathrm{ppm}$ (see Experimental Section). The other hydride signals are due to intermolecular $\mathrm{C}-\mathrm{D}$ activation, followed by $\mathrm{H} / \mathrm{D}$ exchange. Three different isomers are formed by reaction at different positions around the aryl ring and at the methyl group, but no specific assignment is attempted. The formation of the vinyl hydride was favored greatly by the lower temperatures and more viscous medium (Table II). At $188 \mathrm{~K}$ the ratio of vinyl hydride to intermolecular $\mathrm{C}-\mathrm{H}$ activated products was $5.4: 1$, whereas at $273 \mathrm{~K}$ it was only $0.9: 1$. In order to study the effect of solvent viscosity over a narrower range of temperatures, the solvent was changed to hexadecane. $\mathrm{CpIr}(\mathrm{CO})\left(\mathrm{C}_{2} \mathrm{H}_{4}\right)$ was photolyzed $(15 \mathrm{~min}$, $\lambda>328 \mathrm{~nm}$ ) in hexadecane solution at $22^{\circ} \mathrm{C}$ and in a hexadecane solid matrix at $11^{\circ} \mathrm{C}$. The product ratios were monitored by IR spectroscopy. The ratio of vinyl
Table II. Temperature Dependence of the Ratio of Intramolecular to Intermolecular C-H Activation from the Photolysis $(\lambda>290 \mathrm{~nm})$ of $\operatorname{Cp} \operatorname{Ir}(\mathrm{CO})\left(\mathrm{C}_{2} \mathrm{H}_{4}\right)$ in Toluene- $d_{8}$

\begin{tabular}{cccc}
\hline & \multicolumn{2}{c}{ product distribution } & \\
\cline { 2 - 3 } temp $/ \mathrm{K}$ & $\begin{array}{c}\text { vinyl } \\
\text { hydride }\end{array}$ & $\begin{array}{c}\text { tolyl and } \\
\text { benzyl hydrides }\end{array}$ & $\begin{array}{c}\text { viscosity of } \\
\text { toluene } / \mathrm{PP}^{o}\end{array}$ \\
\hline 77 & 1 & 0 & \\
188 & 1 & 0.2 & 4.25 \\
213 & 1 & 0.5 & 2.23 \\
273 & 1 & 1.1 & 0.77
\end{tabular}

a Viscosity values are determined by mathematical interpolation of data given in: CRC Handbook of Chemistry and Physics, 58 th ed., West, R. C., Ed.; CRC Press: Boca Raton, FL, 1977.

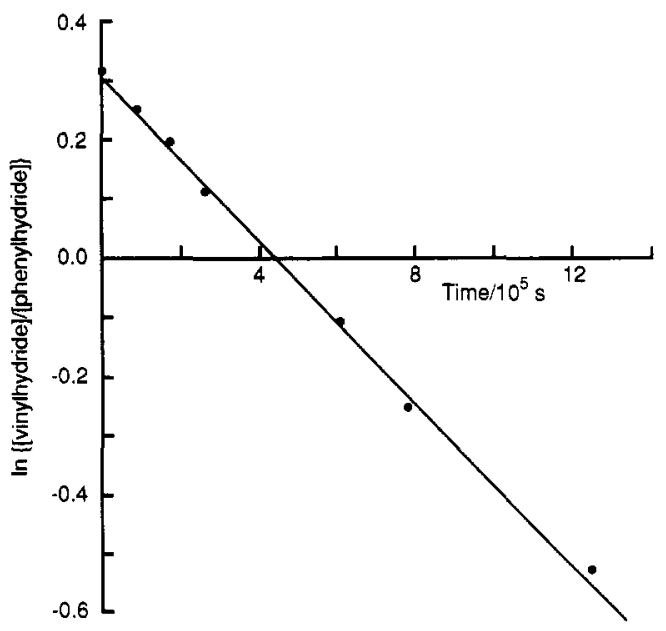

Figure 2. First order plot of $\ln$ ([vinyl hydride]/[pheny] hydride]) vs time for the isomerization of $\mathrm{CpIr}(\mathrm{CO})\left(\mathrm{C}_{2} \mathrm{H}_{3}\right) \mathrm{H}$ to $\mathrm{CpIr}(\mathrm{CO})\left(\mathrm{C}_{2} \mathrm{H}_{4}\right)$ at $284 \mathrm{~K}$ in benzene- $d_{6}$. The symbols represent the experimental points obtained by ${ }^{1} \mathrm{H}$ NMR. The best straight line is shown as a full line.

Table III. Rate Constants for the Thermal Isomerization of $\operatorname{CpIr}(\mathrm{CO})\left(\mathrm{C}_{2} \mathrm{H}_{3}\right)(\mathrm{H})$ to $\mathrm{CpIr}(\mathrm{CO})\left(\mathrm{C}_{2} \mathrm{H}_{4}\right)$ in Benzene- $d_{6}$

\begin{tabular}{cc}
\hline rate constant $/ \mathrm{s}^{-1}$ & temp/K \\
\hline$(6.8 \pm 0.8) \times 10^{-7}$ & 284 \\
$(7.5 \pm 0.8) \times 10^{-6}$ & 298 \\
$(2.4 \pm 0.8) \times 10^{-5}$ & 307 \\
$(5.4 \pm 0.8) \times 10^{-5}$ & 315 \\
$(1.18 \pm 0.08) \times 10^{-4}$ & 323.5 \\
$(2.41 \pm 0.42) \times 10^{-4}$ & 328 \\
$(3.32 \pm 1.03) \times 10^{-4}$ & 332
\end{tabular}

hydride ( $\left.\nu(\mathrm{CO}) 2019 \mathrm{~cm}^{-1}\right)$ :hexadecyl hydride $(\nu(\mathrm{CO}) 2001$ $\mathrm{cm}^{-1}$ ) in solution was 1.7:1 and in the matrix was 2.4:1.

(iv) Thermal Stability of $\mathrm{CpIr}(\mathrm{CO})\left(\mathrm{C}_{2} \mathrm{H}_{3}\right) \mathrm{H}$ and the Rate of Isomerization. $\mathrm{CpIr}(\mathrm{CO})\left(\mathrm{C}_{2} \mathrm{H}_{3}\right) \mathrm{H}$ underwent near complete conversion back to $\operatorname{Cp} \operatorname{Ir}(\mathrm{CO})\left(\mathrm{C}_{2} \mathrm{H}_{4}\right)$ over an 8-day period at $293 \mathrm{~K}$. The rate of the isomerization was monitored at a range of temperatures in benzene- $d_{6}$, by measuring the intensity of the $\operatorname{CpIr}(\mathrm{CO})\left(\mathrm{C}_{2} \mathrm{H}_{3}\right) \mathrm{H}$ hydride resonance in the ${ }^{1} \mathrm{H}$ NMR as a function of time. The measurement was standardized by comparison with the intensity of the $\mathrm{CpIr}(\mathrm{CO})\left(\mathrm{C}_{6} \mathrm{H}_{5}\right) \mathrm{H}$ hydride resonance, which was constant at the range of temperatures used. A first order plot for the decay of vinyl hydride, taken at 284 $\mathrm{K}$, is shown in Figure 2. The rates of reaction (Table III) were used to determine the activation parameters for the thermal isomerization of $\mathrm{CpIr}(\mathrm{CO})\left(\mathrm{C}_{2} \mathrm{H}_{3}\right) \mathrm{H}$ to $\mathrm{CpIr}(\mathrm{CO})$ $\left(\mathrm{C}_{2} \mathrm{H}_{4}\right)$ using the Eyring equation (Figure $\left.3 \mathrm{a}\right), \Delta H^{*}=95$ $\pm 4 \mathrm{~kJ} \mathrm{~mol}^{-1}$ and $\Delta S^{*}=-25 \pm 20 \mathrm{~J} \mathrm{~mol}^{-1} \mathrm{~K}^{-1}$.

(c) $\operatorname{CpIr}\left(\mathrm{C}_{2} \mathrm{H}_{4}\right)_{2}$. It has previously been observed that $\mathrm{CpIr}\left(\mathrm{C}_{2} \mathrm{H}_{4}\right)_{2}$ was isomerized to $\operatorname{CpIr}\left(\mathrm{C}_{2} \mathrm{H}_{4}\right)\left(\mathrm{C}_{2} \mathrm{H}_{3}\right) \mathrm{H}$ on irradiation with UV light in an argon matrix at $12 \mathrm{~K}^{5}$ 

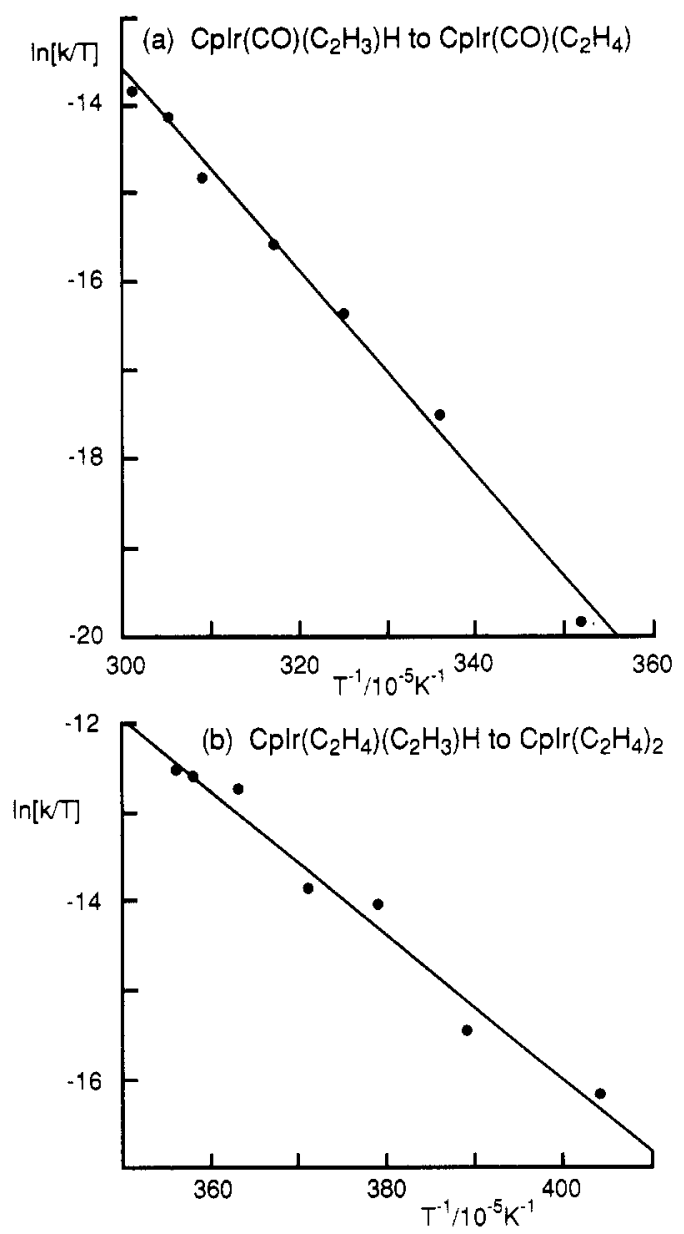

Figure 3. Eyring plots for the temperature dependence of the first order rate constant for the isomerization of (a) $\mathrm{CpIr}$ $(\mathrm{CO})\left(\mathrm{C}_{2} \mathrm{H}_{3}\right) \mathrm{H}$ to $\mathrm{CpIr}(\mathrm{CO})\left(\mathrm{C}_{2} \mathrm{H}_{4}\right)$ in benzene- $d_{6}\left(\Delta H^{*}=95 \pm\right.$ $4 \mathrm{~kJ} \mathrm{~mol}^{-1}$ and $\Delta S^{*}=-25 \pm 20 \mathrm{~J} \mathrm{~mol}^{-1} \mathrm{~K}^{-1}$ ) and (b) $\mathrm{CpIr}$ $\left(\mathrm{C}_{2} \mathrm{H}_{4}\right)\left(\mathrm{C}_{2} \mathrm{H}_{3}\right) \mathrm{H}$ to $\mathrm{CpIr}\left(\mathrm{C}_{2} \mathrm{H}_{4}\right)_{2}$ in toluene- $d_{8}\left(\Delta H^{*}=67 \pm 5\right.$ $\mathrm{kJ} \mathrm{mol}^{-1}$ and $\Delta S^{*}=-60 \pm 19 \mathrm{~J} \mathrm{~mol}^{-1} \mathrm{~K}^{-1}$ ).

When potential ligands such as $\mathrm{N}_{2}$ or $\mathrm{CO}$ were present in the matrix, photolysis of $\mathrm{CpIr}\left(\mathrm{C}_{2} \mathrm{H}_{4}\right)_{2}$ resulted in the formation of $\mathrm{CpIr}\left(\mathrm{C}_{2} \mathrm{H}_{4}\right)\left(\mathrm{C}_{2} \mathrm{H}_{3}\right) \mathrm{H}$ and the substitution products $\mathrm{CpIr}\left(\mathrm{C}_{2} \mathrm{H}_{4}\right)\left(\mathrm{N}_{2}\right)$ and $\mathrm{CpIr}\left(\mathrm{C}_{2} \mathrm{H}_{4}\right)(\mathrm{CO})$. The vinyl hydride was itself photoactive and upon irradiation in an argon matrix underwent further $\mathrm{C}-\mathrm{H}$ activation to form the vinylidene complex $\mathrm{CpIr}\left(\mathrm{CCH}_{2}\right)(\mathrm{H})_{2}$. When CpIr$\left(\mathrm{C}_{2} \mathrm{H}_{4}\right)_{2}$ is irradiated in solid toluene at $77 \mathrm{~K}$, only vinyl hydride formation is observed. ${ }^{5}$

(i) Thermal Stability of $\operatorname{CpIr}\left(\mathrm{C}_{2} \mathrm{H}_{4}\right)\left(\mathrm{C}_{2} \mathrm{H}_{3}\right) \mathrm{H}$ and the Rate of Isomerization. $\mathrm{CpIr}\left(\mathrm{C}_{2} \mathrm{H}_{4}\right)\left(\mathrm{C}_{2} \mathrm{H}_{3}\right) \mathrm{H}$ has the lowest thermal stability of the vinyl hydrides studied here; the complex showed near complete isomerization to $\mathrm{CpIr}$ $\left(\mathrm{C}_{2} \mathrm{H}_{4}\right)_{2}$ in only about $1.2 \mathrm{~h}$ at $273 \mathrm{~K}$.

The vinyl hydride was generated by irradiation of frozen samples of $\mathrm{CpIr}\left(\mathrm{C}_{2} \mathrm{H}_{4}\right)_{2}$ dissolved in toluene- $d_{8}$ at $77 \mathrm{~K}$. The samples were then thawed, and the rates of isomerization were measured using ${ }^{1} \mathrm{H} N \mathrm{NMR}$ at a range of temperatures (Table IV). The measurements were carried out in a manner similar to that used for $\mathrm{CpIr}(\mathrm{CO})\left(\mathrm{C}_{2} \mathrm{H}_{3}\right) \mathrm{H}$, except that the hydride resonance was standardized relative to a grease peak. In order to determine that the vinyl hydride was decaying directly to the $\eta^{2}$-ethene complex, the re-formation of $\mathrm{CpIr}\left(\mathrm{C}_{2} \mathrm{H}_{4}\right)_{2}$ was monitored at one of the temperatures, $269 \mathrm{~K}$. The rate of re-formation of $\mathrm{CpIr}\left(\mathrm{C}_{2} \mathrm{H}_{4}\right)_{2}$ was determined as $(3.08 \pm 0.04) \times 10^{-4} \mathrm{~s}^{-1}$, while that for the loss of $\mathrm{CpIr}\left(\mathrm{C}_{2} \mathrm{H}_{4}\right)\left(\mathrm{C}_{2} \mathrm{H}_{3}\right) \mathrm{H}$ was $(3.19 \pm$
Table IV. Rate Constants for the Thermal Isomerization of $\mathrm{CpIr}\left(\mathrm{C}_{2} \mathrm{H}_{4}\right)\left(\mathrm{C}_{2} \mathrm{H}_{3}\right)(\mathrm{H})$ to $\mathrm{CpIr}\left(\mathrm{C}_{2} \mathrm{H}_{4}\right)_{2}$ in Toluene-d8

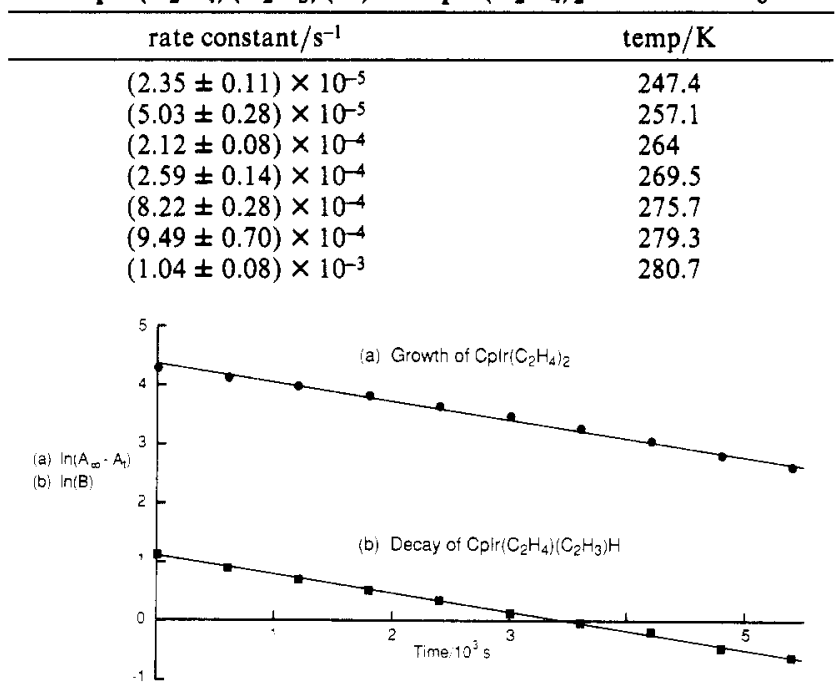

Figure 4. (a) First order plot of $\ln \left(A_{\infty}-A_{t}\right)$ vs time ( $A$ is the integration ratio [ethene complex]/ [grease]) for the isomerization of $\mathrm{CpIr}\left(\mathrm{C}_{2} \mathrm{H}_{4}\right)\left(\mathrm{C}_{2} \mathrm{H}_{3}\right) \mathrm{H}$ to $\mathrm{CpIr}\left(\mathrm{C}_{2} \mathrm{H}_{4}\right)_{2}$ at $269 \mathrm{~K}$ in toluene- $d_{8}$. (b) First order plot of $\ln B$ vs time ( $B$ is the integration ratio [vinyl hydride]/[grease]) for the isomerization of $\mathrm{CpIr}\left(\mathrm{C}_{2} \mathrm{H}_{4}\right)\left(\mathrm{C}_{2} \mathrm{H}_{3}\right) \mathrm{H}$ to $\mathrm{CpIr}\left(\mathrm{C}_{2} \mathrm{H}_{4}\right)_{2}$ at $269 \mathrm{~K}$ in toluene- $d_{8}$.

$0.09) \times 10^{-4} \mathrm{~s}^{-1}$ (Figure 4). These values are the same within experimental error, confirming that $\mathrm{CpIr}\left(\mathrm{C}_{2} \mathrm{H}_{4}\right)$ $\left(\mathrm{C}_{2} \mathrm{H}_{3}\right) \mathrm{H}$ was isomerizing back to $\mathrm{CpIr}\left(\mathrm{C}_{2} \mathrm{H}_{4}\right)_{2}$. An Eyring plot for the isomerization of the $\operatorname{CpIr}\left(\mathrm{C}_{2} \mathrm{H}_{4}\right)\left(\mathrm{C}_{2} \mathrm{H}_{3}\right) \mathrm{H}$ is shown in Figure $3 \mathrm{~b}$. From this plot $\Delta H^{*}$ and $\Delta S^{*}$ for the isomerization are determined as $67 \pm 5 \mathrm{~kJ} \mathrm{~mol}^{-1}$ and -60 $\pm 19 \mathrm{~J} \mathrm{~mol}^{-1} \mathrm{~K}^{-1}$.

(ii) Photolysis of $\mathrm{CpIr}\left(\mathrm{C}_{2} \mathrm{H}_{4}\right)_{2}$ and $\mathrm{PPh}_{3}$. Irradiation $(\lambda>315 \mathrm{~nm})$ of $\mathrm{CpIr}\left(\mathrm{C}_{2} \mathrm{H}_{4}\right)_{2}$ in the presence of a slight excess of $\mathrm{PPh}_{3}$ in $\mathrm{CD}_{3} \mathrm{CN}$ solution caused slow release of ethene and formation of $\mathrm{CpIr}\left(\mathrm{PPh}_{3}\right)\left(\mathrm{C}_{2} \mathrm{H}_{3}\right) \mathrm{H}$ as the principal product. In addition, three minor products are formed: $\mathrm{CpIr}\left(\mathrm{PPh}_{3}\right)\left(\mathrm{C}_{2} \mathrm{H}_{4}\right)$, a complex tentatively identified as $\mathrm{CpIr}\left(\mathrm{PPh}_{3}\right)_{2}$, and a complex formed in $<5 \%$ yield which could only be characterized as an $\left(\eta^{5}\right.$-cyclopentadienyl)iridium hydride. ${ }^{20}$ The reaction was not affected by replacement of $\mathrm{CD}_{3} \mathrm{CN}$ by $\mathrm{CH}_{3} \mathrm{CN}$ or by carrying out the photolysis under $1 \mathrm{~atm}$ of ethene.

When $\mathrm{CpIr}\left(\mathrm{C}_{2} \mathrm{H}_{4}\right)_{2}$ was irradiated in the presence of $\mathrm{PPh}_{3}$ in benzene solution rather than in acetonitrile, two major products were formed: $\mathrm{CpIr}\left(\mathrm{PPh}_{3}\right)\left(\mathrm{C}_{2} \mathrm{H}_{3}\right) \mathrm{H}$ and another hydride together with small amounts of $\mathrm{CpIr}$ $\left(\mathrm{PPh}_{3}\right)\left(\mathrm{C}_{2} \mathrm{H}_{4}\right)$ (preliminary results were reported earlier). ${ }^{2}$ The crude reaction mixture was brominated with $\mathrm{CHBr}_{3}$ at $-78^{\circ} \mathrm{C}$. Following chromatographic purification, two compounds were isolated which were readily identified as $\mathrm{CpIr}\left(\mathrm{PPh}_{3}\right)\left(\mathrm{C}_{2} \mathrm{H}_{3}\right) \mathrm{Br}$ and $\mathrm{CpIr}\left(\mathrm{PPh}_{3}\right)(\mathrm{Ph}) \mathrm{Br}$.

The photolysis reaction was examined using NMR in $\mathrm{C}_{6} \mathrm{D}_{6}$ as a function of time and photolysis wavelength (Table V). At $\lambda>295 \mathrm{~nm}$, the solution darkened and the proportion of phenyl hydride to vinyl hydride increased with time. Irradiation with $\lambda>315 \mathrm{~nm}$ resulted in less decomposition (i.e. the solution darkened much more slowly), so the reaction could be taken to higher conversion. The ratio of the two hydride products remained steady at

(20) $\mathrm{CpIr}\left(\mathrm{PPh}_{3}\right)_{2}$ does not appear to have been characterized previously, but its $\eta^{5}$-indenyl analogue is known. Merola, J. S.; Kamarcik, R. T.; Engen, D. V. J. Am. Chem. Soc. 1986, 108, 329. 
Table V. Product Distribution of the Reaction of $\mathrm{CpIr}\left(\mathrm{C}_{2} \mathrm{H}_{4}\right)_{2}$ and $\mathrm{PPh}_{3}$ in Benzene as a Function of Photolysis Wavelength and Time

\begin{tabular}{cccc}
\hline $\begin{array}{c}\text { photolysis } \\
\text { wavelength } / \mathrm{nm}\end{array}$ & $\begin{array}{c}\text { photolysis } \\
\text { time } / \mathrm{h}\end{array}$ & $\begin{array}{c}\text { \% conversion } \\
\text { to products }\end{array}$ & $\begin{array}{c}\text { ratio of } \\
\mathrm{CpIr}\left(\mathrm{PPh}_{3}\right)\left(\mathrm{C}_{6} \mathrm{D}_{5}\right)(\mathrm{H}) \text { to } \\
\mathrm{CpIr}\left(\mathrm{PPh}_{3}\right)\left(\mathrm{C}_{2} \mathrm{H}_{3}\right)(\mathrm{H})\end{array}$ \\
\hline$>295$ & 1 & 26 & 1.2 \\
& 2.5 & 44 & 1.3 \\
& 3 & 45 & 2.0 \\
$>315$ & 5 & 59 & 2.2 \\
& 6 & 60 & 1.0 \\
& 24 & 65 & 1.0 \\
& 32 & 79 & 1.0 \\
& 55 & 78 & 1.0 \\
& 79 & 89 & 1.0 \\
& 4 & 5 & 0.60 \\
& 20 & 33 & 0.63 \\
& 30 & 45 & 0.63 \\
& 84 & 56 & 0.71
\end{tabular}

1:1. With $\lambda>385 \mathrm{~nm}$ the rate of conversion was inevitably reduced because of low absorption, but the ratio of phenyl hydride to vinyl hydride had now switched to 1:1.6.

A notable feature of this reaction was the occurrence of $\mathrm{H} / \mathrm{D}$ exchange, which could be monitored by using the cyclopentadienyl protons as an internal standard. ${ }^{21}$ Thus photolysis of $\left(\eta^{5}-\mathrm{C}_{5} \mathrm{H}_{5}\right) \operatorname{Ir}\left(\mathrm{C}_{2} \mathrm{H}_{4}\right)_{2}$ with $\mathrm{P}\left(\mathrm{C}_{6} \mathrm{H}_{5}\right)_{3}$ in $\mathrm{C}_{6} \mathrm{D}_{6}$ yielded $\left(\eta^{5}-\mathrm{C}_{5} \mathrm{H}_{5}\right) \operatorname{Ir}\left(\mathrm{PPh}_{3}\right)\left(\mathrm{C}_{6} \mathrm{D}_{5}\right) \mathrm{H}$ (integration of $\mathrm{Cp}$ : hydride $=5: 1$ ). The exchange was not suppressed by use of $\mathrm{P}\left(\mathrm{C}_{6} \mathrm{D}_{5}\right)_{3}$, but reaction of $\left(\eta^{5}-\mathrm{C}_{5} \mathrm{H}_{5}\right) \operatorname{Ir}\left(\mathrm{C}_{2} \mathrm{D}_{4}\right)_{2}$ with $\mathrm{P}\left(\mathrm{C}_{6} \mathrm{H}_{5}\right)_{3}$ in $\mathrm{C}_{6} \mathrm{D}_{6}$ did yield a substantial proportion of deuteride $\left(\eta^{5}-\mathrm{C}_{5} \mathrm{H}_{5}\right) \operatorname{Ir}\left(\mathrm{PPh}_{3}\right)\left(\mathrm{C}_{6} \mathrm{D}_{5}\right) \mathrm{D}(\operatorname{Ir}(\mathrm{Ph}) \mathrm{D}: \operatorname{Ir}(\mathrm{Ph}) \mathrm{H}$ 1:1). However, a similar experiment with $\left(\eta^{5}-\mathrm{C}_{5} \mathrm{H}_{5}\right) \mathrm{Ir}$ $\left(\mathrm{C}_{2} \mathrm{D}_{4}\right)_{2}+\mathrm{P}\left(\mathrm{C}_{6} \mathrm{H}_{6}\right)_{3}$ in $\mathrm{C}_{6} \mathrm{H}_{6}$ yielded no evidence for deuterium in the phenyl hydride product. Thus, hydridic protons in the phenyl hydride product are derived both from benzene and from the original coordinated ethene.

(iii) Thermal Reaction of $\mathrm{CpIr}\left(\mathrm{C}_{2} \mathrm{H}_{4}\right)_{2}$ with $\mathrm{PPh}_{3}$. A control experiment was performed to examine the thermal reaction of $\mathrm{CpIr}\left(\mathrm{C}_{2} \mathrm{H}_{4}\right)_{2}$ with $\mathrm{PPh}_{3}$. At room temperature no reaction took place. However, when CpIr$\left(\mathrm{C}_{2} \mathrm{H}_{4}\right)_{2}$ was heated at $150 \pm 10^{\circ} \mathrm{C}$ for $7 \mathrm{~h}$ in dmso- $d_{6}$ in the presence of $\mathrm{PPh}_{3}$ (70\% excess), $28 \%$ of the starting material was converted to $\mathrm{CpIr}\left(\mathrm{PPh}_{3}\right)\left(\mathrm{C}_{2} \mathrm{H}_{4}\right)$. After longer periods of heating, the compound tentatively identified as $\mathrm{CpIr}\left(\mathrm{PPh}_{3}\right)_{2}$ was also formed.

(iv) Thermal Reaction of $\operatorname{CpIr}\left(\mathrm{C}_{2} \mathrm{H}_{4}\right)\left(\mathrm{C}_{2} \mathrm{H}_{3}\right) \mathrm{H}$ with $\mathbf{P P h}_{3}$. A further control experiment was performed to test the lability of $\mathrm{CpIr}\left(\mathrm{C}_{2} \mathrm{H}_{4}\right)\left(\mathrm{C}_{2} \mathrm{H}_{3}\right) \mathrm{H}$. This vinyl hydride was generated as described above; after photolysis the solution was warmed to $-40^{\circ} \mathrm{C}$, the seal was broken under argon, and a cold solution of $\mathrm{PPh}_{3}$ in toluene- $d_{8}$ was added. The sample was resealed and warmed to room temperature. NMR revealed the presence of $\operatorname{CpIr}\left(\mathrm{C}_{2} \mathrm{H}_{4}\right)_{2}$ but no other CpIr compounds, showing that $\mathrm{CpIr}\left(\mathrm{C}_{2} \mathrm{H}_{4}\right)\left(\mathrm{C}_{2} \mathrm{H}_{3}\right) \mathrm{H}$ had isomerized back to starting material rather than reacting with $\mathrm{PPh}_{3}$.

\section{Discussion}

(a) Competition between Intramolecular and Intermolecular C-H Activation. This study shows that each of the complexes $\mathrm{CpIr}\left(\mathrm{C}_{2} \mathrm{H}_{4}\right)(\mathrm{L})\left(\mathrm{L}=\mathrm{PPh}_{3}, \mathrm{CO}, \mathrm{C}_{2} \mathrm{H}_{4}\right)$ undergoes photoinduced isomerization to the vinyl hydride

(21) Strictly, these experiments measure $H / D$ exchange of the hydride relative to $H / D$ exchange of the cyclopentadienyl protons. An experiment with $\left(\eta^{5}-\mathrm{C}_{6} \mathrm{D}_{5}\right) \operatorname{Ir}\left(\mathrm{C}_{2} \mathrm{H}_{4}\right)_{2}+\mathrm{P}\left(\mathrm{C}_{6} \mathrm{H}_{5}\right)_{3}$ in $\mathrm{C}_{6} \mathrm{D}_{6}$ provides some evidence that such exchange may also contribute.
Scheme II. Matrix Photochemistry of $\operatorname{CpIr}(\mathrm{CO})\left(\mathrm{C}_{2} \mathrm{H}_{4}\right)$

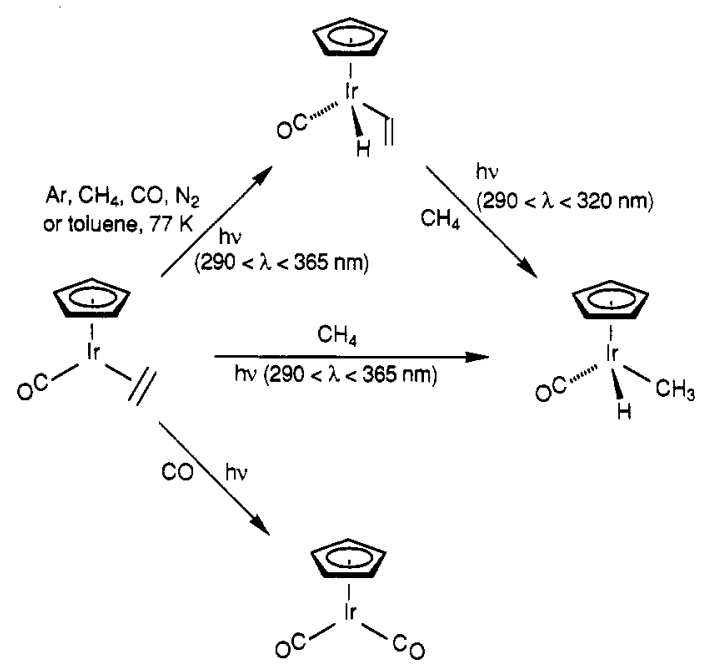

Scheme III. Solution Photochemistry of $\operatorname{CpIr}(\mathrm{CO})\left(\mathrm{C}_{2} \mathrm{H}_{4}\right)$

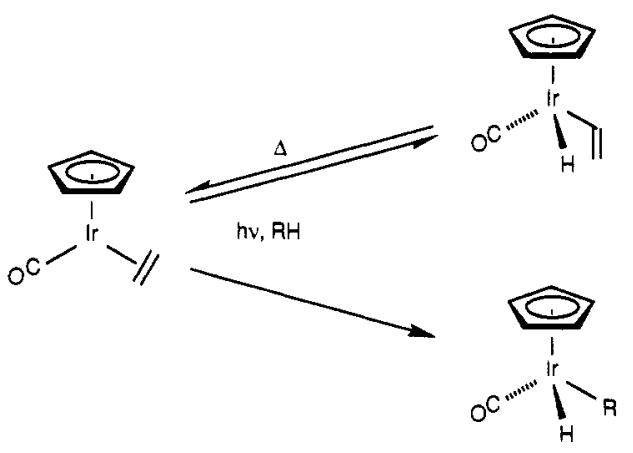

$\mathrm{RH}=$ cyclohexane, benzene, toluene

which, in turn, is converted back to the starting ethene complex thermally (eq 1). In the first two cases, we have also observed that intermolecular $\mathrm{C}-\mathrm{H}$ activation of the solvent competes with intramolecular reaction of coordinated ethene. The reactions of $\mathrm{CpIr}\left(\mathrm{C}_{2} \mathrm{H}_{4}\right)(\mathrm{CO})$ are summarized in Schemes II and III. It might have been expected that $\mathrm{CpIr}\left(\mathrm{C}_{2} \mathrm{H}_{4}\right)\left(\mathrm{PPh}_{3}\right)$ would react to form the orthometalated $\mathrm{CpIr}\left(\mathrm{PPh}_{2} \mathrm{C}_{6} \mathrm{H}_{4}\right) \mathrm{H}$ in preference to insertion into coordinated ethene. We find that little if any orthometalation occurs; the hydride which is tentatively assigned to an orthometalation species accounts for less than $6 \%$ of the product.

The product ratios for intra- and intermolecular $\mathrm{C}-\mathrm{H}$ activation of $\mathrm{CpIr}\left(\mathrm{C}_{2} \mathrm{H}_{4}\right)(\mathrm{L})\left(\mathrm{L}=\mathrm{PPh}_{3}, \mathrm{CO}\right)$ can be controlled by altering the nature of the surrounding media, the excitation wavelength, the temperature, and the ancillary ligand (L). In previous studies on the photoisomerization of metal ethene complexes reported from this laboratory ${ }^{2,5,6}$ we proposed that the reaction mechanism involves a cage-complex intermediate. We argued that the probability of vinyl hydride formation depends on the strength of the metal-ethene interaction in the cage complex and the rigidity of the matrix/solvent cage.

Our present experiments produce much evidence to indicate that the mechanism for $\mathrm{C}-\mathrm{H}$ activation does involve a cage complex. The photoproduct distribution in these complexes can be altered by changing the rigidity of the surrounding medium, the excitation wavelength, and the ancillary ligand. Moreover, when $\operatorname{CpIr}(\mathrm{CO})\left(\mathrm{C}_{2} \mathrm{H}_{4}\right)$ 
is irradiated in a methane matrix it forms $\mathrm{CpIr}(\mathrm{CO})$ $\left(\mathrm{CH}_{3}\right) \mathrm{H}$, but irradiation in an argon matrix does not yield $\mathrm{CpIr}(\mathrm{CO})$. Thus intermolecular $\mathrm{C}-\mathrm{H}$ bond activation can take place without the complete dissociation of the ethene ligand. However, it appears that the mechanism shown in Scheme I is only a partial explanation. Of critical importance are the effects of viscosity and temperature. On the basis of the cage mechanism we had expected that increased viscosity/rigidity of the medium would shift the product distribution toward vinyl hydride. The importance of the rigidity of the surrounding medium is highlighted by the following example: $\mathrm{CpIr}\left(\mathrm{C}_{2} \mathrm{H}_{4}\right)\left(\mathrm{C}_{2} \mathrm{H}_{3}\right) \mathrm{H}$ is only formed in appreciable amounts when photolysis of $\mathrm{CpIr}\left(\mathrm{C}_{2} \mathrm{H}_{4}\right)_{2}$ takes place within a rigid matrix.

In order to study the effect of the viscosity of the surrounding medium in a systematic manner $\mathrm{CpIr}(\mathrm{CO})$ $\left(\mathrm{C}_{2} \mathrm{H}_{4}\right)$ was irradiated under the same conditions in toluene at a range of temperatures. The results of this experiment are shown in Table II, and they clearly show that vinyl hydride formation is favored at lower temperature/more viscous solvent. In order to separate the effects of temperature and viscosity, we examined the photolysis of $\mathrm{Cp} \operatorname{Ir}(\mathrm{CO})\left(\mathrm{C}_{2} \mathrm{H}_{4}\right)$ in liquid and solid hexadecane. The above results show that temperature is more important than viscosity in controlling product distribution, although both play a part. It follows that formation of intermolecular product is a thermally activated process, but it is not clear whether thermal excitation occurs in the ground or excited electronic states. ${ }^{22}$ Thermal excitation of an electronically excited state would only be feasible if that state was long-lived. Therefore, we anticipate that forthcoming TRIR experiments on CpIr(CO) $\left(\mathrm{C}_{2} \mathrm{H}_{4}\right)$ should enable us to distinguish between these two mechanisms.

The nature of the kinetically favored product is also determined by the wavelength of the exciting light, intermolecular $\mathrm{C}-\mathrm{H}$ activation being favored at shorter wavelengths. This behavior is exhibited by $\mathrm{CpIr}(\mathrm{CO})$ $\left(\mathrm{C}_{2} \mathrm{H}_{4}\right)$ both in solution and in a reactive matrix and is consistent with a cage-complex intermediate. Shorter wavelength excitation will result in a vibrationally excited cage complex, from which ethene loss is more favored. This explanation of wavelength dependence is not unique: alternatively, two excited states may be involved. A systematic study of the effect of irradiation wavelength is complicated by the secondary photolysis of the vinyl hydride at shorter wavelengths $(\lambda>290 \mathrm{~nm})$. For comparison, a study on the closely related species $\mathrm{CpRh}$ $(\mathrm{CO})_{2}$ and $\mathrm{Cp} * \mathrm{Rh}(\mathrm{CO})_{2}$ showed that ligand substitution can occur through two mechanisms depending on excitation wavelength, one associative (ring-slip) and one dissociative. ${ }^{23}$ It was proposed that the two mechanisms arose from the population of different excited states.

A change of the ancillary ligand in the metal ethene complex provides another means of controlling the $\mathrm{ki}$ netically favored product. This study investigates the influence of the ligands $\mathrm{PPh}_{3}, \mathrm{CO}$, and $\mathrm{C}_{2} \mathrm{H}_{4}$. When $\mathrm{CpIr}$ $\left(\mathrm{PPh}_{3}\right)\left(\mathrm{C}_{2} \mathrm{H}_{4}\right)$ or $\mathrm{CpIr}(\mathrm{CO})\left(\mathrm{C}_{2} \mathrm{H}_{4}\right)$ is irradiated in benzene$d_{6}$ at room temperature, the ratios of vinyl hydride to phenyl hydride are 3.8:1 and 1:1, respectively. No quantitative measurements have been carried out on the ratio

(22) In an attempt to see if $\mathrm{CpIr}(\mathrm{CO})$ could react with methane thermally at low temperature we formed this species by photolysis of $\mathrm{CpIr}(\mathrm{CO})_{2}$ in a $6 \%$ methane/ $\mathrm{Xe}$ matrix. However annealing the matrix up to $70 \mathrm{~K}$ resulted in no formation of $\mathrm{Cp} \operatorname{Ir}(\mathrm{CO})\left(\mathrm{CH}_{3}\right) \mathrm{H}$.

(23) Drolet, D. P.; Lees, A. J. J. Am. Chem. Soc. 1992, 114, 4186.
Scheme IV. Reaction Mechanism for the Photolysis of $\mathrm{CpIr}\left(\mathrm{C}_{2} \mathrm{H}_{4}\right)_{2}$ and $\mathrm{PPh}_{3}$ in $\mathrm{CH}_{3} \mathrm{CN}$

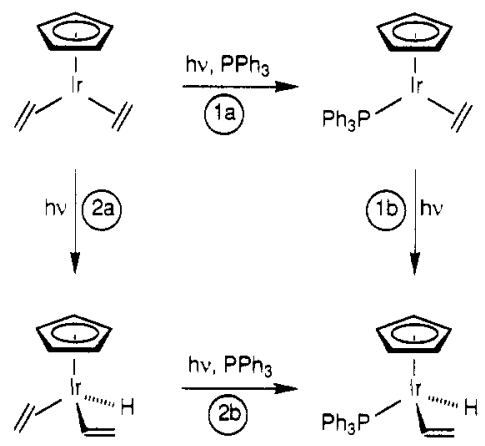

of products from the photolysis of $\mathrm{CpIr}\left(\mathrm{C}_{2} \mathrm{H}_{4}\right)_{2}$ in solution because of the extensive decomposition. Even when CpIr$\left(\mathrm{C}_{2} \mathrm{H}_{4}\right)_{2}$ is irradiated at temperatures sufficiently low for the vinyl hydride to be stable, very little vinyl hydride formation is observed. ${ }^{5}$ At the other extreme, we have observed previously that irradiation of $\mathrm{CpIr}\left(\mathrm{PMe}_{3}\right)\left(\mathrm{C}_{2} \mathrm{H}_{4}\right)$ in solution resulted in the vinyl hydride accounting for greater than $90 \%$ of the products formed. Thus the ancillary ligand is important in determining the stability of the cage complex and hence the proportion of vinyl hydride. In the cases discussed, the strength of the metalethene interaction increases in the order $\mathrm{C}_{2} \mathrm{H}_{4}<\mathrm{CO}<$ $\mathrm{PPh}_{3}<\mathrm{PMe}_{3}$.

(b) Thermal Reversal: Vinyl Hydride to Ethene Complex. The nature of the ancillary ligand plays an important role in determining the size of the thermal barrier to the isomerization of vinyl hydride back to the $\eta^{2}$-ethene complex. The complex $\mathrm{CpIr}\left(\mathrm{PPh}_{3}\right)\left(\mathrm{C}_{2} \mathrm{H}_{3}\right) \mathrm{H}$ has the largest barrier to thermal reconversion, with a rate constant of $(7.0 \pm 0.5) \times 10^{-6} \mathrm{~s}^{-1}$ at $391 \pm 2 \mathrm{~K}$. CpIr(CO) $\left(\mathrm{C}_{2} \mathrm{H}_{3}\right) \mathrm{H}$ and $\mathrm{CpIr}\left(\mathrm{C}_{2} \mathrm{H}_{4}\right)\left(\mathrm{C}_{2} \mathrm{H}_{3}\right) \mathrm{H}$ are more thermally labile and have rates of isomerization estimated to be ca. $8.2 \times 10^{-2}$ and $1.2 \times 10^{-1} \mathrm{~s}^{-1}$ at $392 \mathrm{~K}$, respectively. The enthalpies of activation for the isomerization of $\mathrm{CpIr}(\mathrm{CO})$ $\left(\mathrm{C}_{2} \mathrm{H}_{3}\right) \mathrm{H}$ and $\mathrm{CpIr}\left(\mathrm{C}_{2} \mathrm{H}_{4}\right)\left(\mathrm{C}_{2} \mathrm{H}_{3}\right) \mathrm{H}$ are calculated to be 95 \pm 3 and $67 \pm 5 \mathrm{~kJ} \mathrm{~mol}^{-1}$, respectively. The barrier to isomerization increases with ancillary ligand in the order $\mathrm{C}_{2} \mathrm{H}_{4}<\mathrm{CO}<\mathrm{PPh}_{3}$. The enthalpy of activation for the thermal isomerization of $\mathrm{Cp} * \operatorname{Ir}\left(\mathrm{PMe}_{3}\right)\left(\mathrm{C}_{2} \mathrm{H}_{3}\right) \mathrm{H}$ to $\mathrm{Cp} * \mathrm{Ir}$ $\left(\mathrm{PMe}_{3}\right)\left(\mathrm{C}_{2} \mathrm{H}_{4}\right)$ in cyclohexane- $d_{12}$ is even higher: $145 \pm 5$ $\mathrm{kJ} \mathrm{mol}{ }^{-1} \cdot 1$

(c) Reaction of $\mathrm{CpIr}\left(\mathrm{C}_{2} \mathrm{H}_{4}\right)_{2}$ with $\mathrm{PPh}_{3}$ : Combined Substitution and Oxidative Addition. The major product of the photochemical reaction of $\mathrm{CpIr}\left(\mathrm{C}_{2} \mathrm{H}_{4}\right)_{2}$ with $\mathrm{PPh}_{3}$ in $\mathrm{CH}_{3} \mathrm{CN}$ is $\mathrm{CpIr}\left(\mathrm{PPh}_{3}\right)\left(\mathrm{C}_{2} \mathrm{H}_{3}\right) \mathrm{H}$. In addition, small amounts of $\mathrm{CpIr}\left(\mathrm{PPh}_{3}\right)\left(\mathrm{C}_{2} \mathrm{H}_{4}\right)$ are formed. This reaction provides a much simpler route to $\mathrm{CpIr}\left(\mathrm{PPh}_{3}\right)\left(\mathrm{C}_{2} \mathrm{H}_{3}\right) \mathrm{H}$ than isomerization of $\mathrm{CpIr}\left(\mathrm{PPh}_{3}\right)\left(\mathrm{C}_{2} \mathrm{H}_{4}\right)$, since the latter requires a laborious synthesis. A control experiment shows that there is no thermal reaction of $\operatorname{CpIr}\left(\mathrm{C}_{2} \mathrm{H}_{4}\right)_{2}$ and $\mathrm{PPh}_{3}$ until a high temperature is attained. Two plausible routes for the formation of the vinyl hydride may be envisaged (Scheme IV). In the first (1), substitution by $\mathrm{PPh}_{3}$ precedes isomerization to the vinyl hydride. In the second (2), isomerization of $\mathrm{CpIr}\left(\mathrm{C}_{2} \mathrm{H}_{4}\right)_{2}$ precedes substitution. ${ }^{24}$ We know already that $\mathrm{CpIr}\left(\mathrm{PPh}_{3}\right)\left(\mathrm{C}_{2} \mathrm{H}_{4}\right)$ is isomerized to the vinyl hydride photochemically, so the first route is fully consistent with our observations. A control exper-

(24) Since we do not observe $\mathrm{CpIr}\left(\mathrm{CH}_{3} \mathrm{CN}\right)\left(\mathrm{C}_{2} \mathrm{H}_{4}\right)$ (in contrast to the rhodium analogue), we do not consider it as a possible intermediate. 
Scheme V. Reaction Mechanism for the Photolysis of $\mathrm{CpIr}\left(\mathrm{C}_{2} \mathrm{H}_{4}\right)_{2}$ and $\mathrm{PPh}_{3}$ in Benzene

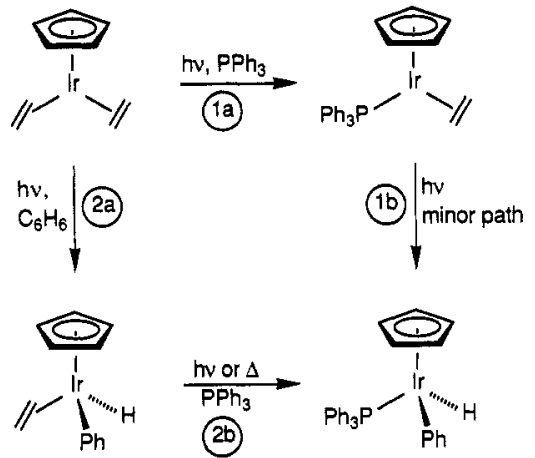

Scheme VI. Cage-Complex Reaction Mechanism Incorporating a Thermal Step to Intermolecular C-H Bond Activation

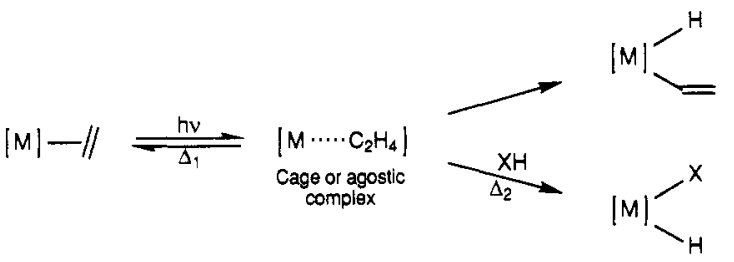

iment demonstrates that $\operatorname{CpIr}\left(\mathrm{C}_{2} \mathrm{H}_{4}\right)\left(\mathrm{C}_{2} \mathrm{H}_{3}\right) \mathrm{H}$ does not undergo thermal substitution by $\mathrm{PPh}_{3}$, so both routes require two photons. However, we have also established that $\mathrm{CpIr}\left(\mathrm{C}_{2} \mathrm{H}_{4}\right)\left(\mathrm{C}_{2} \mathrm{H}_{3}\right) \mathrm{H}$ is not formed in significant quantities at room temperature and is short-lived above $5{ }^{\circ} \mathrm{C}$. We conclude, therefore, that route 1 must be dominant.

When the reaction between $\mathrm{CpIr}\left(\mathrm{C}_{2} \mathrm{H}_{4}\right)_{2}$ and $\mathrm{PPh}_{3}$ is performed in benzene, $\mathrm{CpIr}\left(\mathrm{PPh}_{3}\right)(\mathrm{Ph}) \mathrm{H}$ is formed as an additional product. Again, there are two reasonable routes for its formation (Scheme V): either substitution followed by solvent oxidative addition (1) or vice versa (2). Since we observe $\mathrm{CpIr}\left(\mathrm{PPh}_{3}\right)\left(\mathrm{C}_{2} \mathrm{H}_{4}\right)$, we know that route 1 can contribute. However, we have examined the photolysis of $\mathrm{CpIr}\left(\mathrm{C}_{2} \mathrm{H}_{4}\right)\left(\mathrm{PPh}_{3}\right)$ and find that we obtain drastically less phenyl hydride compared to vinyl hydride than when we irradiate $\mathrm{CpIr}\left(\mathrm{C}_{2} \mathrm{H}_{4}\right)_{2}$ in benzene with $\mathrm{PPh}_{3}$. It follows that route 2 must act as the major source of $\mathrm{CpIr}\left(\mathrm{PPh}_{3}\right)$ $(\mathrm{Ph}) \mathrm{H}$. At present, we have not sought to establish whether the second step of route 2 is photochemically or thermally induced. When the reaction mixture is irradiated with a short wavelength cutoff $(\lambda>295 \mathrm{~nm})$, secondary photolysis of $\mathrm{CpIr}\left(\mathrm{PPh}_{3}\right)\left(\mathrm{C}_{2} \mathrm{H}_{3}\right) \mathrm{H}$ may also alter the product distri- bution. In addition to the steps of Scheme IV and V, these reactions are complicated by $\mathrm{H} / \mathrm{D}$ exchange processes involving the solvent, the coordinated ethene, and possibly the cyclopentadienyl protons. Such exchange is also evident in the photoinduced reaction of $\operatorname{CpIr}(\mathrm{CO})\left(\mathrm{C}_{2} \mathrm{H}_{4}\right)$ with $\mathrm{C}_{6} \mathrm{D}_{6}$. Catalytic $\mathrm{H} / \mathrm{D}$ exchange has been studied in detail elsewhere. ${ }^{25}$ The mechanism of the process observed here has not been established.

\section{Conclusions}

(1) Intramolecular $\mathrm{C}-\mathrm{H}$ activation of ethene competes with intermolecular activation of solvent in the photochemistry of $\operatorname{CpIr}(\mathrm{L})\left(\mathrm{C}_{2} \mathrm{H}_{4}\right)\left(\mathrm{L}=\mathrm{PPh}_{3}, \mathrm{CO}, \mathrm{C}_{2} \mathrm{H}_{4}\right)$ complexes.

(2) The ancillary ligand has a major effect in determining (i) the product ratio and (ii) the kinetic stability of the vinyl hydride with respect to the ethene complexes.

(3) The product ratios are also dependent on the temperature, the rigidity of the surrounding medium, and the photolysis wavelength.

(4) The product distributions are consistent with the formation of an intermediate cage complex [CpIr(L) $\cdots \mathrm{C}_{2} \mathrm{H}_{4}$ ]. Insertion of ethene competes with displacement by solvent. The temperature dependence of the product distribution probably arises from the barrier to displacement of the ethene in the cage by the solvent (Scheme VI). This thermal step was overlooked in our previously proposed mechanism (Scheme I). However, thermal excitation of a long-lived electronically excited state has not yet been rigorously excluded. Changes in the ancillary ligand, L, favor ethene insertion if the incage $\sigma$-interaction is strengthened. The strength of the metal-vinyl $\sigma$ bond of the product is probably influenced by a change in $\mathrm{L}$ in the same way.

(5) Photolysis of $\mathrm{CpIr}\left(\mathrm{C}_{2} \mathrm{H}_{4}\right)_{2}$ in the presence of $\mathrm{PPh}_{3}$ exploits these reactions to give a "one-pot" route to $\mathrm{CpIr}$ $\left(\mathrm{PPh}_{3}\right)\left(\mathrm{C}_{2} \mathrm{H}_{3}\right) \mathrm{H}$ and $\mathrm{CpIr}\left(\mathrm{PPh}_{3}\right)(\mathrm{Ph}) \mathrm{H}$.

Acknowledgment. We are pleased to acknowledge the support of The Royal Society, The European Commission, NATO, SERC, and British Gas. We are grateful to Prof. M. Poliakoff and Dr. M. George for carrying out the TRIR experiments and to Prof. J. Shapley for revealing results prior to publication.

\section{OM930116M}

(25) Grebenik, P. D.; Green, M. L. H.; Izquierdo, A.; Mtetwa, V. S. B.; Prout, K. J. Chem. Soc. Dalton Trans. 1987, 9. 